\title{
A numerical technique to simulate display pixels based on electrowetting
}

\author{
Ivo Roghair ${ }^{1,2} \cdot$ Michiel Musterd ${ }^{3,4} \cdot$ Dirk van den Ende $^{1}$ - Chris Kleijn ${ }^{4}$. \\ Michiel Kreutzer $^{3} \cdot$ Frieder Mugele $^{1}$
}

Received: 5 July 2014 / Accepted: 31 March 2015 / Published online: 17 April 2015

(C) The Author(s) 2015. This article is published with open access at Springerlink.com

\begin{abstract}
We present a numerical simulation technique to calculate the deformation of interfaces between a conductive and non-conductive fluid as well as the motion of liquid-liquid-solid three-phase contact lines under the influence of externally applied electric fields in electrowetting configuration. The technique is based on the volume of fluid method as implemented in the OpenFOAM framework, using a phase fraction parameter to track the different phases. We solve the combined electrohydrodynamic problem by coupling the equations for electric effects-Gauss's law and a charge transport equation-to the Navier-Stokes equations of fluid flow. Specifically, we use a multi-domain approach to solving for the electric field in the solid and liquid dielectric parts of the system. A Cox-Voinov boundary condition is introduced to describe the dynamic contact angle of moving contact lines. We present several benchmark problems with analytical solutions to validate the simulation model. Subsequently, the model is used to study the dynamics of an electrowetting-based display pixel. We demonstrate good qualitative agreement between simulation results of the opening and closing of a pixel with experimental tests of the identical reference geometry.
\end{abstract}

Ivo Roghair

i.roghair@tue.nl

1 Physics of Complex Fluids, University of Twente, Enschede, The Netherlands

2 Chemical Process Intensification, Eindhoven University of Technology, Eindhoven, The Netherlands

3 Product and Process Engineering, Delft University of Technology, Delft, The Netherlands

4 Transport Phenomena, Delft University of Technology, Delft, The Netherlands
Keywords Electrowetting - OpenFOAM - Pixel · Cox-Voinov $\cdot$ Contact line

\section{List of symbols}

Greek symbols

$\alpha \quad$ Phase fraction

$\beta \quad$ Ratio of viscosities

$\gamma \quad$ Interface tension $(\mathrm{N} / \mathrm{m})$

$\delta \quad$ Weighting parameter (value fraction)

$\Delta n \quad$ Distance from cell centre to cell face (m)

$\varepsilon_{0} \quad$ Electric constant $(\mathrm{F} / \mathrm{m})$

$\varepsilon_{\mathrm{d}} \quad$ Relative electric permittivity of dielectric

$\eta \quad$ Electrowetting number

$\theta \quad$ Contact angle $\left(^{\circ}\right)$

$\kappa \quad$ Interface curvature $\left(\mathrm{m}^{-1}\right)$

$\mu \quad$ Fluid viscosity ( $\mathrm{Pa} \mathrm{s}$ )

$\rho \quad$ Density $\left(\mathrm{kg} / \mathrm{m}^{3}\right)$

$\rho_{\mathrm{E}} \quad$ Electric charge density $\left(\mathrm{C} / \mathrm{m}^{3}\right)$

$\sigma \quad$ Electric conductivity $(\mathrm{S} / \mathrm{m})$

$\phi \quad$ Electric potential (V)

$\phi_{\mathrm{m}} \quad$ Mass flux $\left(\mathrm{kg} / \mathrm{m}^{2} / \mathrm{s}\right)$

\section{Roman symbols}

Bo $\quad$ Bond number

$C_{\gamma} \quad$ Interface compression coefficient

$\mathrm{Ca}$ Capillary number

$\mathrm{Ca} a_{\mathrm{E}} \quad$ Electrocapillary number

$d \quad$ Dielectric layer thickness (m)

$D$ Aspect ratio

$\vec{E} \quad$ Electric field $(\mathrm{V} / \mathrm{m})$

$\vec{F} \quad$ Force $(\mathrm{N})$

$\vec{g} \quad$ Gravitational acceleration $\left(\mathrm{m} / \mathrm{s}^{2}\right)$

$\vec{I} \quad$ Identity matrix

$\vec{n} \quad$ Unit normal 
$p \quad$ Pressure $\left(\mathrm{N} / \mathrm{m}^{2}\right)$

$Q \quad$ Ratio of permittivities

$R \quad$ Ratio of conductivities

$R_{\mathrm{d}} \quad$ Radius (m)

$S_{\mathrm{f}} \quad$ Cell surface area $\left(\mathrm{m}^{2}\right)$

$t \quad$ Time (s)

$\overrightarrow{\vec{T}} \quad$ Maxwell stress tensor $\left(\mathrm{N} / \mathrm{m}^{2}\right)$

$\vec{u} \quad$ Fluid velocity $(\mathrm{m} / \mathrm{s})$

$\vec{u}_{c} \quad$ Compression velocity $(\mathrm{m} / \mathrm{s})$

$\vec{x} \quad$ Position $(\mathrm{m})$

$y \quad$ Position $(\mathrm{m})$

\section{Introduction}

Applying electric fields is one of the most powerful techniques to manipulate small amounts of conductive liquids such as water in non-conductive ambient environments such as air or oil. The electric fields lead to a Maxwell stress pulling on the liquid-liquid interface. In recent years, electrowetting (Mugele and Baret 2005) has evolved into the arguably most popular technique for electrical manipulation of liquids in microfluidics with a wide range of applications including lab-on-a-chip systems (Fair 2007), optofluidics (Berge and Perseux 2000; Kuiper and Hendriks 2004; Krupenkin et al. 2003; Murade et al. 2011, 2012), energy harvesting (Krupenkin and Taylor 2011) and display technology (Hayes and Feenstra 2003; Sun and Heikenfeld 2008). Electrowetting on dielectric (EWOD) involves a specific geometry with a thin dielectric coating separating an electrode from the conductive liquid sitting on top of it (Fig. 1).

In many applications, only the motion of the drop on a global scale much larger than the thickness of the dielectric coating is of interest. In this case, the action of the Maxwell stress can be reduced to a net force per unit length that pulls on the three-phase contact line and therefore reduces

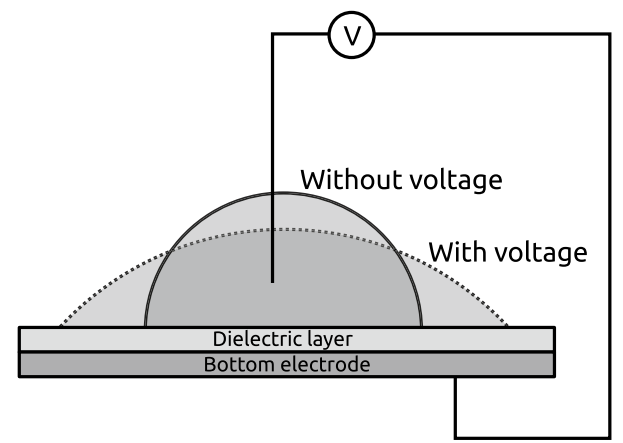

Fig. 1 Sketch of a classic electrowetting on dielectric setup. A sessile droplet on a dielectric surface with immersed electrode has a large contact angle if no voltage is applied, which decreases with increasing voltage the apparent contact angle $\theta$ (Jones et al. 2001; Buehrle et al. 2003). Provided that the applied voltage is not too high, the contact angle reduction follows the electrowetting or Young-Lippmann equation:

$\cos \theta=\cos \theta_{Y}+\frac{\varepsilon_{0} \varepsilon_{\mathrm{d}}}{2 d \gamma} \phi^{2}=\cos \theta_{Y}+\eta$

In this equation, the apparent contact angle $\theta$ can be described using Young's contact angle $\theta_{Y}$, and the electrowetting number $\eta=\frac{\varepsilon_{0} \varepsilon_{\mathrm{d}}}{2 d \gamma} \phi^{2}$, which is equal to the ratio of the strength of electrostatic energy and surface tension $\gamma$. The dielectric constant of the insulating layer is given by $\varepsilon_{\mathrm{d}}$, its thickness by $d$, and the applied potential is given by $\phi$. Close to the three-phase contact line, however, local electric fringe fields give rise to a strong deformation and high curvature of the liquid-liquid interface resulting from the balance of the local Maxwell stress and Laplace pressure at any point on the interface. As a result, Young's angle is still maintained on a local scale $\ll d$ (Buehrle et al. 2003; Mugele and Buehrle 2007).

In the present article, we describe the development and validation of a simulation technique that allows for a dynamic calculation of the liquid distribution upon applying an external voltage simultaneously resolving both local and global electrostatic field and fluid distributions in complex geometries that involve electrodes as well as topographically patterned solid dielectrics. We apply our technique to simulate the specific situation of a pixel within an electrowetting-based display with a geometry similar to the original design by Hayes and Feenstra (2003). This concept involves a liquid container (initially square or rectangular, but keeping the possibility to extend to arbitrary shapes) filled with a perfect dielectric, coloured oil, with on top an electrically conducting aqueous phase. Electrodes are placed at the bottom and the top of the pixel. The bottom electrode is separated from the oil using a thin, hydrophobic, insulating layer of amorphous fluoropolymer (Fig. 2).

By applying an electric potential to the bottom electrode, while keeping the top electrode at ground voltage, the emerging electric field will interact with the oil-aqueous interface. The interface bends down due to the electrohydrodynamic force and creates a three-phase contact line. From that moment, electrowetting takes place and further moves the three-phase contact line (and thus the oil phase) to the edges of the pixel, revealing the pixel bottom. The advantage of this technique over conventional LCD displays is that the ambient light reflects on the surface of the display, instead of requiring an energy- and space-consuming backlight.

The creation of a design and testing of microfluidic electrohydrodynamic (EHD) devices requires a lot of time-consuming experimental work. For this reason, many researchers have used numerical simulations for their investigations. 
Fig. 2 Exploded view of a typical structure of an electrowetting pixel. From top to bottom top electrode, spacing volume (filled with electrolyte and coloured oil), pixel walls, dielectric layer and bottom electrode

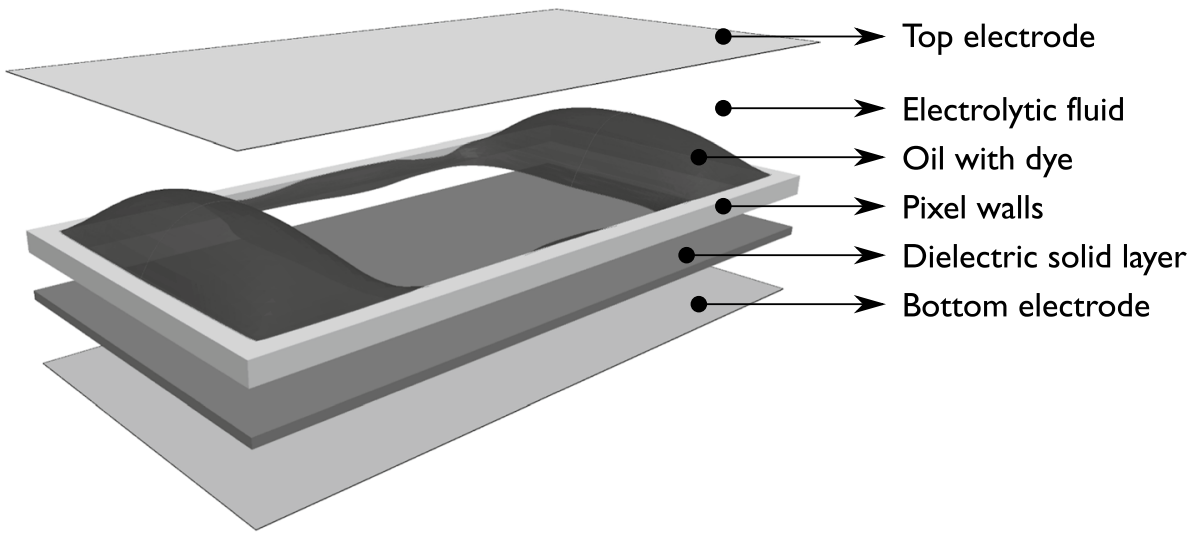

For instance, $\mathrm{Ku}$ et al. (2011) are among the first who attempted the simulation of different electrode patterns for use in electrowetting-based displays. Many studies have been devoted to electrohydrodynamics, for instance Tomar et al. (2007), Bjørklund (2009), López-Herrera et al. (2011) and Lima and d'Ávila (2013). While electrohydrodynamics is an important aspect of electrowetting, the effect of the three-phase contact line has not been studied in these works. For instance, while the Gerris EHD code due to López-Herrera et al. (2011) provides an accurate framework for EHD calculations alone, the explicit definition of the contact angle in three dimensions is not possible.

EWOD has been studied by others using different approaches. Arzpeyma et al. (2008) and Keshavarz-Motamed et al. (2010) study the actuation of a droplet onto an electrode using a volume of fluid (VOF) method. They implemented a two-way coupling scheme of the electric potential field and the VOF solver. The effect of electrowetting has been modelled by modifying the contact angle of the drop using the Young-Lippmann equation with local electric potential. Similarly, lattice-Boltzmann simulations have been performed by Aminfar and Mohammadpourfard $(2009,2012)$ in which both the electric field and the contact angle of moving and merging droplets have been resolved. In contrast to the VOF models mentioned earlier, these lattice-Boltzmann simulations derive the contact angle using adjusted surface tension coefficients that change with the applied electric potential. Arzpeyma et al. (2008) found a rather sharp transition between the equilibrium (zero voltage) contact angle and the contact angle on top of the electrode. Therefore, the approach of Dolatabadi et al. (2006) and Clime et al. (2010a, b) may be justified; their simulations of droplet movement are performed using a positiondependent contact angle, hereby omitting the need to solve for the electric field.

While the method used by Clime et al. (2010a, b) and Dolatabadi et al. (2006) has its physical justification for purely electrowetting cases (e.g. Buehrle et al. (2003), Mugele and Buehrle (2007), the display pixel case requires a combination of electrohydrodynamic and electrowetting modelling. As long as the electrolytic aqueous phase does not have a contact point with the dielectric layer, the problem is purely electrohydrodynamic since there is no threephase contact line. When the three-phase contact line is formed, due to electrohydrodynamic retraction of the oil, the problem becomes of the electrowetting type. At this time, the electric field within the dielectric layer on which the droplet is deposited must be considered. Resolving the electric field in both the fluid phase and the solid phase is deemed essential for the purpose of simulating electrowetting pixels; when the oil has retracted, the two electrodes are separated by nothing than an electrolytic fluid, which short-circuits the system. A model for the display pixels hence requires accounting for the electric field distribution, as demonstrated in our earlier work (Manukyan et al. 2011) and explained in the modelling paper by Oh et al. (2012).

Hong et al. (2008), Drygiannakis et al. (2009), and Pooyan and Passandideh-Fard (2012) have simulated EWOD including the electric field strength in the dielectric layer and provide a thorough explanation of the numerical technique. Drygiannakis et al. (2009) intention is to investigate contact angle saturation due to dielectric breakdown with this model. However, it is not clear whether these methods also include pure electrohydrodynamic effects.

For the current investigation, the desired model has a number of requirements which have, combined, not been found in the literature. The numerical model that is capable of simulating a wide range of electrowetting devices would ideally consist of the following aspects:

- Multiphase flow solver: To distinguish between the electrolyte and the oil phase, a multiphase flow technique that can handle deformable interfaces including surface tension and topological transitions is preferred.

- Dynamic contact angle model: The dynamic behaviour of the system should include a physically correct implementation of the dynamic contact angle model, for 
instance the Cox-Voinov model. This allows the simulation of a velocity-dependent contact angle.

- Electrostatic field solver: The solution of a Laplace equation (Gauss's law) to determine the electric field is required, taking into account local variations of the electric permittivity.

- Include electric field inside dielectric layer: Since the electric field is applied over both the spacing volume (including electrolyte and oil) and the solid dielectric layer, the electric field needs to be solved in a coupled fashion in both domains.

- Capability of simulating perfect dielectric, perfect conducting and leaky dielectric compounds.

- Ability of simulating arbitrary domain shapes

The creation of an electro-hydrodynamic model is described in the following section, including the modelling of multiple domains, simulating both the fluid flow region and the dielectric region in a two-way coupled manner. The next section discusses the verification of different aspects of the model, by comparison with analytical solutions. The model will consequently be used to simulate pixels based on electrowetting, including both the closing and opening behaviour. Finally, we present a discussion on the numerical method and on the obtained results and concluding remarks.

\section{Model development}

In this section, the governing equations are outlined. While the discussion on the fluid flow and electric field effects is based on our earlier work (Roghair et al. 2013), this work extends the model with appropriate Cox-Voinov (Cox 1986; Voinov 1976) contact angle boundary conditions and the incorporation of finite-size solid parts.

We have chosen to build our model using the OpenFOAM framework. OpenFOAM is an open-source software package capable of numerically solving a wide range of computational fluid dynamics (CFD)-related problems. Thanks to its open nature, we are able to incorporate the electrostatic field equations and the interaction of the electric field with the fluid-fluid interface into the existing framework. We use the source code of 'interFoam' as a base model (using version 2.1.1). While the basics of this model are described below, an extensive evaluation and verification of this multiphase flow solver is given in the work of Deshpande et al. (2012) and references therein.

\subsection{Fluid flow}

The fluid flow field $\vec{u}$ is solved via the incompressible Navier-Stokes equations, given in Eq. 2 and 3:

$$
\begin{aligned}
\frac{\partial \rho \vec{u}}{\partial t}+\nabla \cdot(\rho \vec{u} \vec{u})= & -\nabla p+\nabla \cdot\left[\mu\left(\nabla \vec{u}+\nabla \vec{u}^{\mathrm{T}}\right)\right] \\
& +\rho \vec{g}+\vec{F}_{\gamma}+\vec{F}_{\mathrm{E}}
\end{aligned}
$$

$\nabla \cdot \vec{u}=0$

In these equations, $\rho$ and $\mu$ represent the macroscopic density and viscosity, respectively, $p$ represents the pressure, and $\vec{u}$ is the velocity. The additional terms $\vec{F}_{\gamma}$ and $\vec{F}_{\mathrm{E}}$ account for the forces due to surface tension and the electric field (the latter will be discussed in the next section). The multi-phase flow method incorporated in OpenFOAM is based on the volume of fluid (VOF) method (Deshpande et al. 2012), allowing to simulate fluid-fluid flows with dynamic interfaces including surface tension forces. The VOF method is a widely used technique, seen in many different implementations (see e.g. Hirt and Nichols 1981; Popinet 2009; Baltussen et al. 2014), and while the implementations may differ largely, the technique generally describes the fluids and the interfaces by accounting for a phase fraction field variable $\alpha$. This variable ranges between 0 (accounting for one phase) and 1 (accounting for another phase), while any value in between indicates the transition region, i.e. the presence of an interface. The source forces $\vec{F}_{\gamma}$ and $\vec{F}_{\mathrm{E}}$ are mapped to the cell volumes that contain the interface as volumetric forces (even though they represent surface forces). At each time step, the phase fraction field variable is advected with the fluid flow via Eq. 4:

$\frac{\partial \alpha}{\partial t}+\nabla \cdot(\alpha \vec{u})=0$

The advantages of VOF are intrinsic volume conservation and possibility of topological changes, e.g. break-up or merging of dispersed elements, but the OpenFOAM implementation lacks a sharp interface as seen in other methods, e.g. front-tracking (Unverdi and Tryggvason 1992; Dijkhuizen et al. 2010) and Level-Set (Sussman et al. 1994, 1999), or other VOF implementations that use a geometric interface reconstruction (Popinet 2009; Baltussen et al. 2014). Instead, OpenFOAM makes use of a compression algorithm that limits the numerical interface smearing. The origin of this technique is found in the work of Ubbink and Issa (1999) (compressive interface capturing scheme for arbitrary meshes, CICSAM) and Muzaferija and Perić (1998) (high resolution interface capturing scheme, HRIC), where effectively an artificial compression term $-\nabla \cdot\left(\alpha(1-\alpha) \vec{u}_{c}\right)$ is added to Eq. 4 so that numerical diffusion is counteracted:

$\frac{\partial \alpha}{\partial t}+\nabla \cdot(\alpha \vec{u})-\nabla \cdot\left(\alpha(1-\alpha) \vec{u}_{c}\right)=0$

with artificial compression velocity 
$\vec{u}_{c}=\vec{n}_{\mathrm{f}} \min \left[C_{\gamma} \frac{\left|\phi_{\mathrm{m}}\right|}{\left|S_{\mathrm{f}}\right|}, \max \left(\frac{\left|\phi_{\mathrm{m}}\right|}{\left|S_{\mathrm{f}}\right|}\right)\right]$

where $\vec{n}_{\mathrm{f}}$ is the normal vector of the cell surface, $\phi_{\mathrm{m}}$ is the mass flux, $S_{\mathrm{f}}$ is the cell surface area, and $C_{\gamma}$ is an adjustable coefficient, the value of which can be set between 0 and 4 (see also Hoang et al. 2013) who explain the compression technique in more detail and have performed a number of tests on the effectiveness of the $C_{\gamma}$ coefficient and show the benefits of an additional smoother operator to limit parasitic currents that emerge when using larger compression values $\left(1<C_{\gamma} \leq 4\right)$. For systems dominated by capillary forces, a moderate setting $C_{\gamma}=1$ has been shown to give the best balance between sharpening and induced parasitic currents. The special discretization scheme for this advection equation uses MULES (multidimensional universal limiter with explicit solution) to ensure boundedness of the phase fraction variable (see Sussman et al. 1994; Zalesak 1979).

In the computational cells containing the interface $(0<\alpha<1)$, the field variables representing the density and viscosity are obtained via weighted arithmetic averaging with the phase fraction:

$\rho=\rho_{1} \alpha+\rho_{2}(1-\alpha)$

$\mu=\mu_{1} \alpha+\mu_{2}(1-\alpha)$

The surface tension force $\vec{F}_{\gamma}$ is obtained using the continuum surface force (CSF) model due to Brackbill et al. (1992). The volumetrically distributed surface tension force is only active in cells containing the interface, formulated as in Eq. 9:

$\vec{F}_{\gamma}=\gamma \kappa(\nabla \alpha)$

The local curvature of the interface $\kappa$ is obtained from the divergence of the surface normals, which can be obtained via the phase fraction field distribution:

$\kappa=-\nabla \cdot \vec{n}=\nabla \cdot\left(\frac{\nabla \alpha}{|\nabla \alpha|}\right)$

\subsection{Electric equations}

To account for the electrostatic field, Gauss's law is solved, relating the electric field with free electric charges in the domain:

$\nabla \cdot(\varepsilon \nabla \phi)=-\rho_{\mathrm{E}}$

in which $\varepsilon$ denotes the electric permittivity, $\phi$ is the electric potential, and $\rho_{\mathrm{E}}$ is the electric charge density, which are all represented as volumetric scalar quantities. The Laplacian discretization has been performed using the Gauss linear corrected scheme and solved by a preconditioned conjugate gradient method, which are available by default in OpenFOAM. The charge density is advected with the fluid flow and conducted according to the fluid conductivity using the following transport equation (López-Herrera et al. 2011):

$\frac{\partial \rho_{\mathrm{E}}}{\partial t}+\nabla \cdot\left(\rho_{\mathrm{E}} \vec{u}\right)=\nabla \cdot(\sigma \nabla \phi)$

Here, $\sigma$ denotes the conductivity of the fluid (Saville 1997). The charge transport equation is solved by a preconditioned biconjugate gradient method. The charge density is advected by straightforward advection with the fluid flow using a Van Leer total variation diminishing scheme and not with the MULES scheme used for the phase fraction variable. The MULES scheme clips the fluxes of the phase fraction variable when the receiving cell goes out of bounds (i.e. phase fractions larger than 1 or smaller than 0). For the charge density, this is not deemed a problem, and conventional transportation fluxes are used. This means that, as a result, the charges may not be advected consistently to the phase fraction parameter. This may introduce spurious currents, as the forces exerted on the electric charges may not exactly coincide with the position of the interface. This is, however, merely a possibility, we have not found any significant influences of these currents, nor evidence for their existence in the simulations presented in this work.

The electric relaxation time $t_{\mathrm{e}}=\varepsilon / \sigma$ presents a time step constraint (López-Herrera et al. 2011); along with the Courant criterium $\|\vec{u}\| \Delta t / \Delta x$ which is kept below 0.1 , the global simulation time step is adaptively set according to:

$t_{\mathrm{sim}}=0.9 \min \left[\min \left\{\frac{\varepsilon_{1}}{\sigma_{1}}, \frac{\varepsilon_{2}}{\sigma_{2}}\right\}, 0.1 \frac{\Delta x}{\|\vec{u}\|}\right]$

In the transition region, the permittivity is obtained via arithmetic averaging, and the conductivity is obtained via harmonic averaging, both weighted with the phase fraction:

$\frac{1}{\sigma}=\frac{\alpha}{\sigma_{1}}+\frac{(1-\alpha)}{\sigma_{2}}$

$\varepsilon=\alpha \varepsilon_{1}+(1-\alpha) \varepsilon_{2}$

Tomar et al. (2007) found more accurate results using the weighted harmonic averaging approach as opposed to the weighted harmonic mean. We have not found a significant influence of the interpolation method for the permittivity, but using the weighted harmonic averaging for the conductivity creates a much steeper transition of the conductivity field compared to arithmetic averaging and keeps electric charges outside of the lower-conductivity domain. This prevents the charges from getting 'trapped' inside a zero-conductivity liquid. This effect becomes less important with smaller cell sizes (also seen by López-Herrera et al. 2011), since the charges are physically a quantity on the interface only which are numerically represented as a volumetric 
scalar. Finally, we couple the electrostatic field to the interface via the Maxwell stress tensor $\vec{T}$, which is incorporated via $\vec{F}_{\mathrm{E}}$ in the momentum (prediction) equations. It is computed just before the momentum matrix is constructed and added as an explicit source term to the momentum matrix. The pressure correction is automatically corrected for this term in the momentum matrix (not involving any modification to the pressure computation). The Maxwell stress tensor is evaluated as a volumetric, cell-centred tensor field, and its divergence using the standard OpenFOAM divergence operator results in a cell-centred electric force contribution:

$\vec{F}_{\mathrm{E}}=\nabla \cdot \overrightarrow{\vec{T}}$

$\overrightarrow{\vec{T}}=\varepsilon\left(\vec{E} \vec{E}-\frac{|\vec{E}|^{2}}{2} \overrightarrow{\vec{I}}\right)$

The electric force $F_{\mathrm{E}}$ thus also is a continuum force, which only (significantly) acts near the interface; only in this region, the change in the electric field strength (due to the change in electric properties) is such that the Maxwell stress tensor is of significant size to yield an effective force on the fluid. The electric field $\vec{E}$ is computed by taking the gradient of the electric potential:

$\vec{E}=-\nabla \phi$

With these equations included, the model is able to solve both dielectric and fully conductive liquids, and any combination of the two. The electric equations are solved on the same computational mesh as the fluid flow equations. We have also taken care that the dynamic meshing feature delivered with interFoam is conserved, which allows to refine the mesh according to a user-defined criterium (typically near the interface). For each variable, boundary conditions can be set, such as Neumann (zero flux) and Dirichlet (fixed value) or combinations of the two.

\subsection{Dielectric solid layer}

It is important to simulate the electric field in the dielectric solid layer. The fluid flow on top is solved dynamically, and the fluid-fluid configuration is used to perform an electrostatic computation resolving the electric field and charge distribution. The electric field strength in the solid layer is important as well, since it is affected by the fluid flow on top of it. Since no fluid flow is allowed inside the solid region, a distinction has to be made between two domains, which are to be coupled using their common electrical variable.
OpenFOAM offers a basic framework to set up a multiregion domain, allowing for the simulation of adjacent regions which are governed by different sets of equations, but where the common variables are communicated via the common boundary between two regions. The governing equation for the dielectric layer is Gauss's law (Eq. 11), since the dielectric layer contains no free charges. In principle, it should be possible to simulate the migration of charges via the solid phase as well. However, this is not necessary here, because for common electrowetting problems, a support material with strong electrically insulating properties is used. Hence, the fluid flow domain and the solid phase domain have been coupled via a single boundary variable for the electric potential, $\phi$. In OpenFOAM, the common way to couple the boundary values and gradients between two domains is to use the mixed boundary condition. This procedure allows to set the value and the gradient of a variable via a weighting parameter $\delta$, that blends the value and gradient at the corresponding boundary:

$\phi_{i, \text { wall }}=\delta_{i} \phi_{j, \text { cell }}+\left(1-\delta_{i}\right)\left(\phi_{i, \text { cell }}+\frac{\mathrm{d} \phi_{i}}{\mathrm{~d} n} \Delta n\right)$

The gradient term $\frac{\mathrm{d} \phi_{i}}{\mathrm{~d} n}$ is set to zero when the mixed boundary condition is used as a coupling boundary condition; hence, only the cell-centred electric potential on both sides is taken into account:

$\phi_{i, \text { wall }}=\delta_{i} \phi_{j, \text { cell }}+\left(1-\delta_{i}\right) \phi_{i, \text { cell }}$

This condition is evaluated at both sides of the coupled boundary, so that $i$ indicates the solid and $j$ indicates the liquid, or vice versa. The weighting parameter (termed valueFraction) is defined by the electric permittivities of the two domains and the cell size in the normal direction at the coupled boundary, $\Delta n$ :

$\delta_{i}=\left(1-\delta_{j}\right)=\frac{\varepsilon_{j} / \Delta n_{j}}{\left(\varepsilon_{j} / \Delta n_{j}+\varepsilon_{i} / \Delta n_{i}\right)}$

The choice of Eq. 21 makes sure that Eq. 19 is numerically equivalent on both sides of the boundary as explained in the OpenFOAM source 'solidWallMixedTemperatureCoupled' in "derivedFvPatchFields". In the numerical procedure, Gauss's law is solved in all domains separately, using Eq. 19 to obtain a boundary value for the potential. In order to make sure that the boundary values are consistent throughout different domains, a number of Newton iterations are performed alternating over the domains.

\subsection{Dynamic contact angle}

OpenFOAM natively offers a dynamic contact angle boundary condition that adapts the contact angle to the interface normal velocity $u$. While moving contact lines 
are normally problematic in sharp interface techniques, an advantage of the numerical smearing of the interface mentioned earlier is that it automatically resolves the wellknown stress singularity for moving contact lines. In this model, the contact angle varies symmetrically around the equilibrium contact angle for outward normal (positive) and inward normal (negative) velocities with a given upper and lower bound (through a hyperbolic tangent). It is, however, well known that the dynamic contact angle does not vary symmetrically with positive and negative velocities. Rather it has been shown for sufficiently small contact angles that the dynamic contact angle follows the Cox-Voinov equation (Cox 1986; Voinov 1976) of Eq. 22. A more extensive review of validity can be found in Bonn et al. (2009) and Snoeijer and Andreotti (2013).

$\theta^{3}=\theta_{Y}^{3}+9 \frac{\mu u}{\gamma} \ln \frac{x_{\max }}{x_{\min }}$

where $\mu$ is the viscosity of the more viscous liquid and $x_{\max }$ and $x_{\min }$ are upper and lower cut-off lengths which physically relate to the droplet size and a molecular size respectively, which have been set to $x_{\max }=4.874 \times 10^{-4}$ and $x_{\min }=2 \times 10^{-8} \mathrm{~m}$. Note that only the order of magnitude of these parameters has significant influence, not the exact value, due to the natural logarithm. The contact angle itself is forced to the prescribed value through an appropriate correction to the surface force, $F_{\gamma}$. We implement the CoxVoinov equation in the existing framework of the dynamic contact angle boundary condition, thus allowing a more physical contact angle boundary condition that moreover includes the effects of the fluid properties on the dynamic behaviour. Our implementation does not capture contact line pinning due to microscopic, i.e. subpixel, heterogeneities, but for electrowetting pixels great care is taken to avoid those so this will not limit applicability.

\section{Validation}

Various parts of the newly implemented model are validated through a series of cases, which have been inspired by the cases worked by López-Herrera et al. (2011). The validation cases all solve the entire set of equations. When only a specific aspect is verified (e.g. the electric equations), the other equations (e.g. the two-phase flow) are set up without a driving force.

\subsection{Validation of electric equations}

The first validation considers the solution of the electric equations (Gauss's law and charge transport equation) in one dimension. Consider a domain that is equally divided between two immiscible fluids (interface in the domain centre), both of which can be either perfect dielectric or conducting. A potential difference is applied over the two boundaries of the domain, resulting in an electric field through the fluids which can be described analytically according to the permittivity ratio $Q=\varepsilon_{1} / \varepsilon_{2}$ and conductivity ratio $R=\sigma_{1} / \sigma_{2}$. Three cases can be distinguished; two conductive fluids, two dielectric fluids and a combination of a dielectric and a conductive fluid. Figure $3 \mathrm{com}-$ pares the analytical (exact) solution with the simulation outcome for these cases using 100 cells between the two electrodes, showing good correspondence between theory and simulation Table 1 lists the analytical solutions.

\subsection{Validation of multi-region implementation}

Additionally, a validation case is set up using the multiregion approach, dividing a domain into three equal parts (solid, fluid 1 and fluid 2), all modelled as perfect dielectrics. The electric field $\frac{\mathrm{d} \phi}{\mathrm{d} y}$ is inversely proportional to the

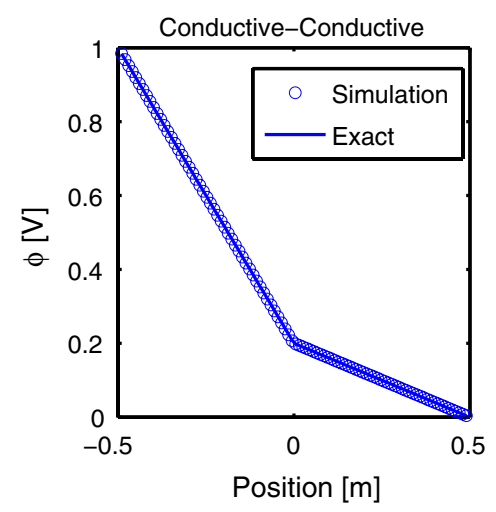

Fig. 3 Comparison between analytical and simulation results for a domain equally divided between two fluids (interface at the kink) and a potential difference of $1 \mathrm{~V}$ is applied. The electric field strength for
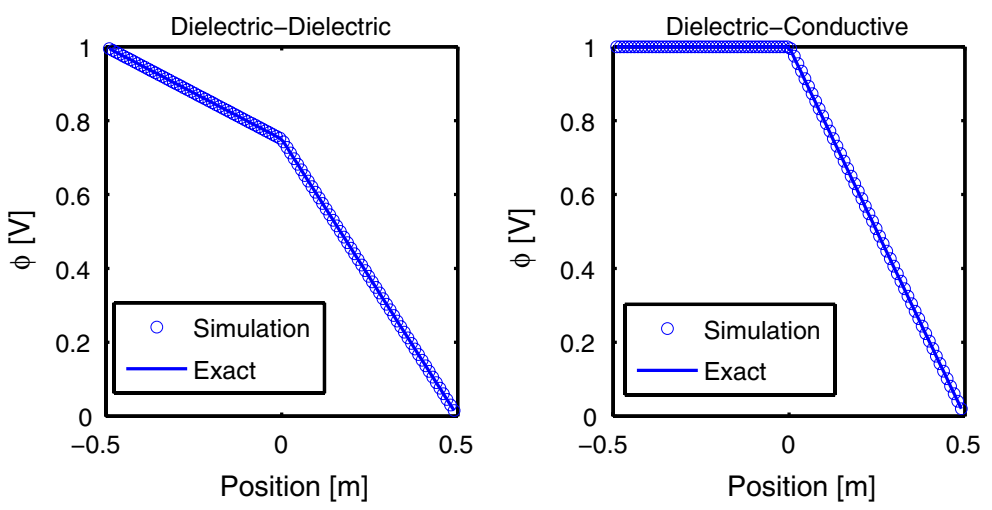

the different cases depends on the following parameters-Left conductivity ratio $R=0.25$; Centre permittivity ratio $Q=3$; Right Solution depends on position only 
Table 1 Exact solutions for the two-phase planar layer cases following López-Herrera et al. (2011)

\begin{tabular}{llll}
\hline & $\begin{array}{l}\text { Conductive- } \\
\text { conductive }\end{array}$ & $\begin{array}{l}\text { Dielectric- } \\
\text { dielectric }\end{array}$ & $\begin{array}{l}\text { Dielectric- } \\
\text { conductive }\end{array}$ \\
\hline Property & $R=\frac{\sigma_{1}}{\sigma_{2}}=0.25$ & $Q=\frac{\varepsilon_{1}}{\varepsilon_{2}}=3$ & $1 / R=0, \quad Q=3$ \\
Fluid 1 & $\phi_{1}^{\text {exact }}=\frac{-2 y+R}{1+R}$ & $\phi_{1}^{\text {exact }}=\frac{-2 y+Q}{1+Q}$ & $\phi_{1}^{\text {exact }}=1$ \\
Fluid 2 & $\phi_{2}^{\text {exact }}=\frac{R(-2 y+1)}{1+R}$ & $\phi_{2}^{\text {exact }}=\frac{Q(-2 y+1)}{1+Q}$ & $\phi_{2}^{\text {exact }}=1-2 y$ \\
\hline
\end{tabular}

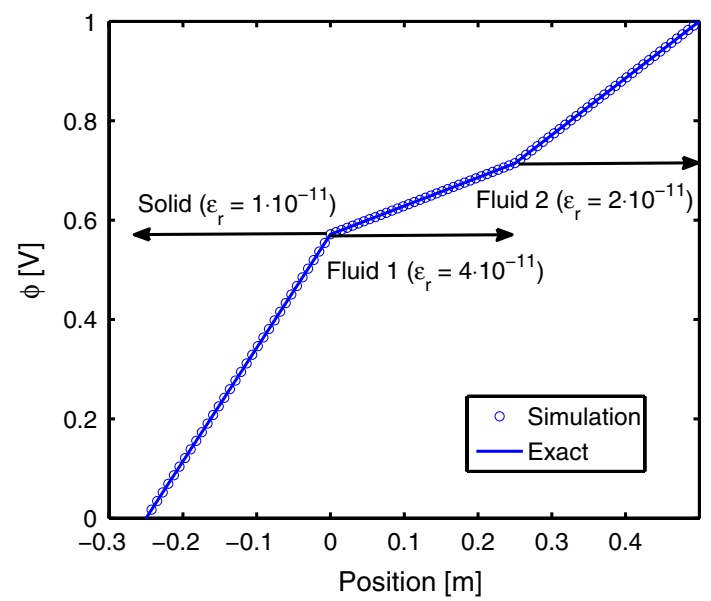

Fig. 4 Comparison between exact and simulation results for a domain equally divided in a solid and two liquid parts with different permittivities

electric permittivity of one medium and scales with the total potential drop over the domain, so that electric flux over all transitions (solid-fluid domain and fluid-fluid interface) is retained:

$\varepsilon_{\text {solid }}(\nabla \phi)_{\text {solid }}=\varepsilon_{\text {fluid } 1}(\nabla \phi)_{\text {fluid } 1}=\varepsilon_{\text {fluid } 2}(\nabla \phi)_{\text {fluid } 2}$

Figure 4 shows the parameters used for this case and shows that the coupling boundary is implemented adequately due to good agreement of the analytical results are given in Table 2.

\subsection{Gaussian charge bump}

In order to verify the correct implementation of conductivity, a Gaussian charge bump is centred in a domain filled with a single conductive fluid, using gradient-free walls.
The charges repel each other and cause the initial profile to decay according to the following relation:

$\rho_{\mathrm{E}}(\vec{x}, t)=\rho_{\mathrm{E}}(\vec{x}, t=0) e^{-\frac{\sigma t}{\varepsilon}}$

with

$\rho_{\mathrm{E}}(\vec{x}, t=0)=\frac{\exp \left(-\frac{\|\vec{x}\|^{2}}{2 a^{2}}\right)}{a \sqrt{2 \pi}}$

The parameter $a$ is used to set the width of the bell, and $\vec{x}$ represents the position. Figure 5a shows a good correspondence of the simulation result, at different times.

\subsection{Charged cylinder case}

Another case, verifying the implementation of the charge transport equation in combination with Gauss's law, is the use of a conducting cylinder immersed in a perfect dielectric medium. When the cylinder is charged, the charges repel and travel to the edge of the cylinder. The charges generate an electric field in the dielectric medium, of which the magnitude is compared with analytical relations in Fig. $5 b$.

\subsection{Rate of convergence}

Apart from these verification tests, the rate of convergence of the numerical solvers with respect to the mesh size has been investigated. The solution to Gauss's law is investigated using the dielectric-dielectric planar layer case. The $L^{\infty}$ error norm shows a rate of convergence of unity, whereas the $L^{2}$ norm decreases with a rate of about 0.5 . The electric charge transport equation has a rate of convergence of 2 based on the $L^{2}$ error norm, as shown in Fig. 6 .

\subsection{Validation of fully-coupled implementation}

The coupling of the electric field and the interface, via the Maxwell stress tensor (Eq. 17), is verified by comparing the deformation of a droplet subjected to an electric field to results from the literature. The general aspects of this case have been discussed in many other works in the literature (Taylor 1966; Tomar et al. 2007; López-Herrera et al. 2011; Lima and d'Ávila 2013). We simulated a drop with radius $R_{\mathrm{d}}=0.1 \mathrm{~m}$, in an axisymmetrical domain. Further settings

Table 2 Exact solution to the three-phase planar layer case with solid, fluid 1 and fluid 2 on the domain $y \in[-0.25,0.5]$ with the interfaces at $y=0, y=0.25$

\begin{tabular}{llll}
\hline & Solid & Fluid 2 & Fluid 1 \\
\hline Electric permittivity & $\varepsilon_{\mathrm{S}}=1 \times 10^{-11}$ & $\varepsilon_{2}=4 \times 10^{-11}$ & $\varepsilon_{1}=2 \times 10^{-11}$ \\
Solution & $\phi^{\text {exact }}=\frac{y+0.25}{\varepsilon_{\mathrm{s}} \varepsilon_{\text {tot }}}$ & $\phi^{\text {exact }}=\frac{0.25}{\varepsilon_{\mathrm{s}} \varepsilon_{\text {tot }}}+\frac{y}{\varepsilon_{2} \varepsilon_{\text {tot }}}$ & $\phi^{\text {exact }}=\frac{0.25}{\varepsilon_{\mathrm{s}} \varepsilon_{\text {tot }}}+\frac{0.25}{\varepsilon_{2} \varepsilon_{\text {tot }}}+\frac{y-0.25}{\varepsilon_{1} \varepsilon_{\text {tot }}}$ \\
\hline
\end{tabular}




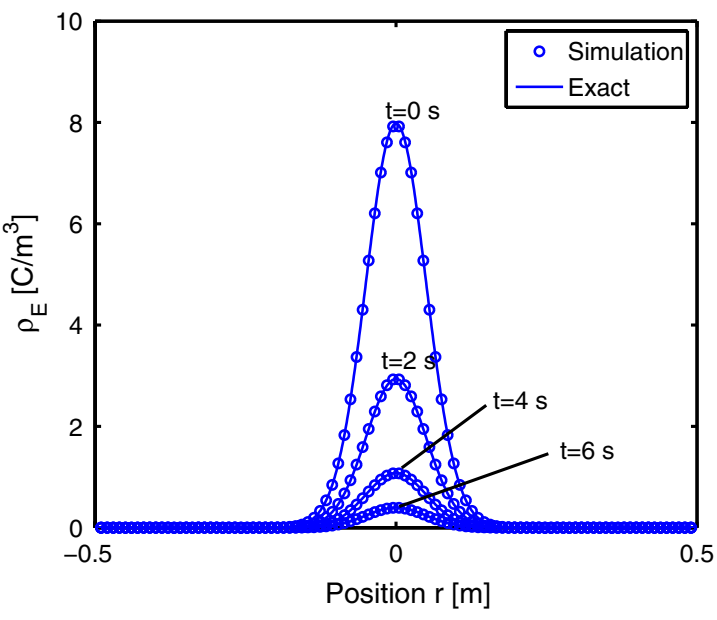

(a)

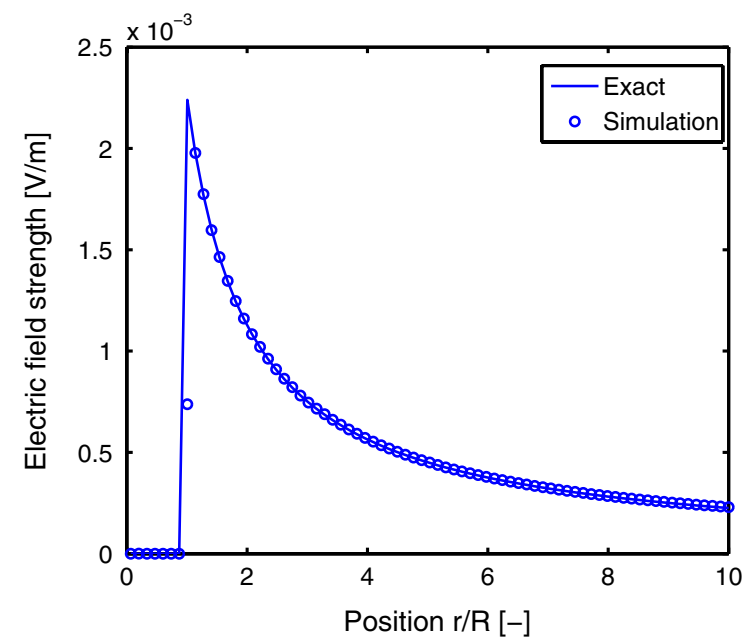

(b)

Fig. 5 Charge transport verification cases using analytical and simulation results. a Shows the temporal evolution of a Gaussian charge bump in a conductive liquid and $\mathbf{b}$ shows the effective electric potential due to charges in a conducting cylinder surrounded by a perfect dielectric

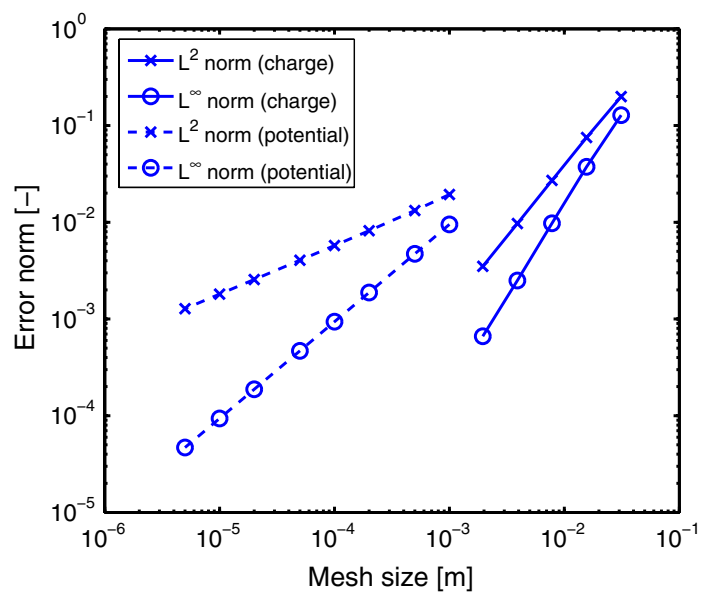

Fig. 6 Rate of convergence of the electric charge and electric potential based on different error norms. The convergence rate for the charge transport equation was obtained from the Gaussian charge bump simulation. For the electric potential, the solution of Gauss's law on the planar layer (dielectric-dielectric) case was used

where a ratio of conductivities of $R=\sigma_{\text {drop }} / \sigma_{\text {ambient }}=2.5$, a ratio of permittivities of $Q=\varepsilon_{\text {drop }} / \varepsilon_{\text {ambient }}=2$ and a viscosity ratio $\beta=\mu_{\mathrm{drop}} / \mu_{\text {ambient }}$ of unity. The governing parameter in this system is the electric capillary number:

$C a_{\mathrm{E}}=\frac{E_{\infty}^{2} R_{\mathrm{d}} \varepsilon_{\text {ambient }}}{\gamma}$

Taylor (1966) has shown that for small deformations, the aspect ratio $D$ is given by:

$D=\frac{9}{16} \frac{C a_{\mathrm{E}}}{(2+R)}\left[1+R^{2}-2 Q+\frac{3}{5}(R-Q) \frac{2+3 \beta}{1+\beta}\right]$
The aspect ratio is defined as $D=\frac{a-b}{a+b}$, where $a$ is the radius parallel and $b$ is the radius perpendicular to the electric field. For different electric capillary numbers, the deformation has been obtained from the simulations and compared to Eq. 27 (see Fig. 7). While the simulation results show a fair correspondence to the analytical correlation, deviations start to become quite large above $C a_{\mathrm{E}}=1$ as can be expected from the prerequisite that deformations should not be too large.

\subsection{Contact angle validation}

Finally, we validate the contact angle boundary condition by comparing with sliding drop experiments from literature (Le Grand et al. 2005). The model system consists of a sliding droplet on a plane inclined at different angles $\alpha$. Due to the motion of the droplet, the contact angle at the front of the droplet $\theta_{\mathrm{f}}$ increases, while the angle at the back $\theta_{\mathrm{b}}$ decreases. This results in a force directed uphill which scales with $\cos \theta_{\mathrm{b}}-\cos \theta_{\mathrm{f}}$ and effectively works as an added friction. We analyse the non-dimensional sliding velocity, $C a=\mu_{\mathrm{drop}} u / \gamma$ as a function of the tilt angle which is non-dimensionalized as $B o \sin \alpha$, where the Bond number $B o=\rho V^{2 / 3} g / \gamma$ quantifies the relative importance of gravity over surface tension. Figure 8 shows a good correspondence of the simulation results with the experiments by Le Grand et al. for two different grid sizes $(\Delta x)$. The significant overestimation of the sliding velocity in case of a constant contact angle boundary condition is also indicated, emphasizing the necessity of using the Cox-Voinov boundary conditions for dynamic systems. 
Fig. 7 a The simulated deformation of a conducting drop immersed in a conducting liquid in an electric field is compared to the relation due to Taylor (1966). b A snapshot of the deformed drop at $C a_{\mathrm{E}}=1$, including flow field and droplet outline. The bottom axis provides rotational symmetry. Colours indicate the electric field strength (colour figure online)

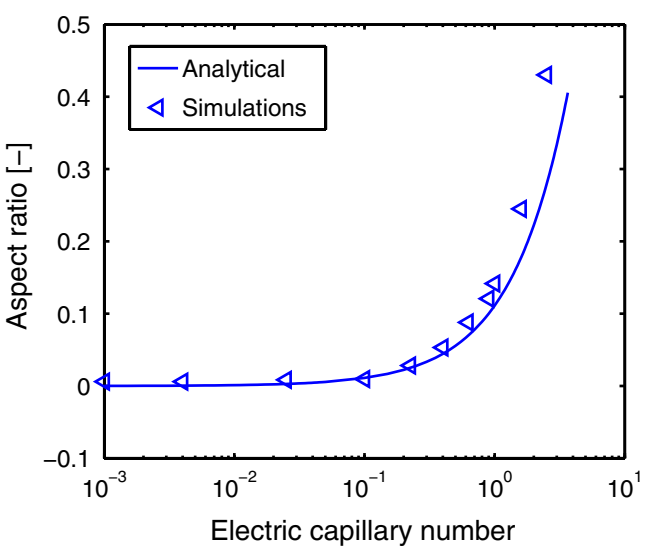

(a)

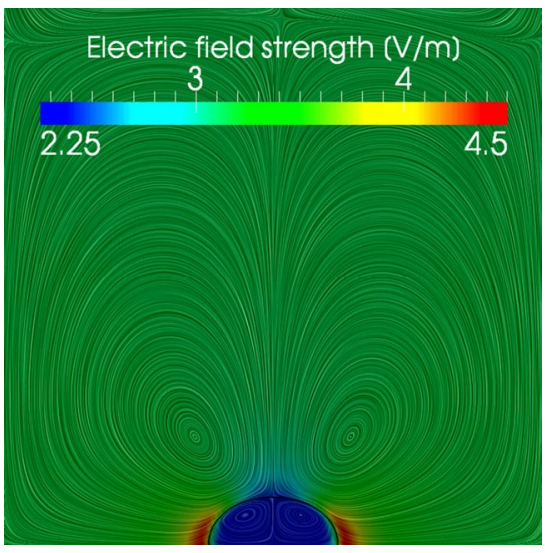

(b)

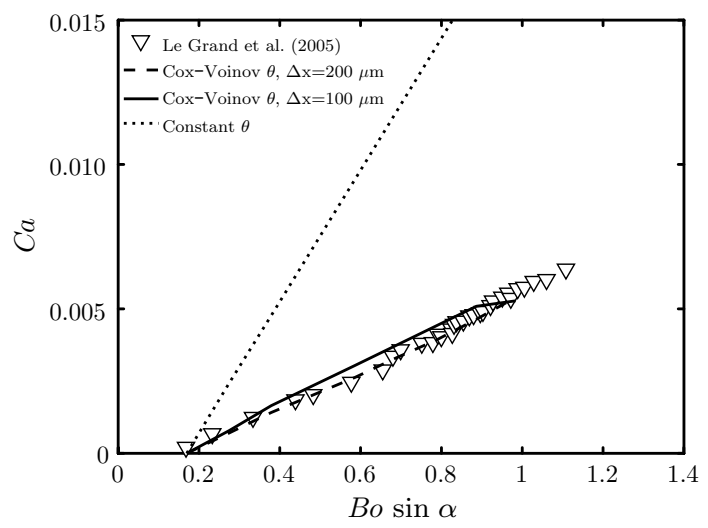

Fig. 8 Comparison of the non-dimensional sliding velocity $\mathrm{Ca}$ of a droplet on an incline at angle $\alpha$. For both small and large grid cells $(\Delta x)$, the simulations with Cox-Voinov BC accurately predict the experiments

\section{Electrowetting pixel simulations}

The dynamic oil switching behaviour (e.g. response times, electric field distribution in the oil) has been investigated for pixel closing and pixel opening. In this work, we keep the geometry of the pixel fixed and discuss cases in which we vary as parameters the volume of oil and the applied voltage. Both electrodes extend to the entire length and width of the pixel, both in the simulations and in comparative experiments. Note that in practice, the electrodes would cover only part of the bottom and could even have a pattern to aid the quick withdrawal of oil (e.g. Ku et al. 2011). Alternative pixel designs, internal structures and tuning of the physical parameters lie outside of the scope of this study.

From a top-view perspective, the area enclosed by the pixel walls is rectangular, being twice as long as it is wide. The walls are $4 \mu \mathrm{m}$ high and are placed right on top of the bottom boundary of the fluid domain. While these walls are impermeable and serve as a physical boundary for the oil in a pixel, the domain boundaries above the walls up to the top of the domain (which may vary from 20 to 100 but is typically $54 \mu \mathrm{m}$ high) are open so that the aqueous fluid may freely flow in and out.

Numerical simulations are performed using the electrowetting pixel geometry as shown in Fig. 2, using a mesh as shown in Fig. 9 and boundary conditions as listed in Table 3. The base geometry was set up using the blockMesh tool provided with OpenFOAM, which stacks three hexagonal regions with a Cartesian mesh, all of which share a width of 24 cells, and 96 cells in length:

1. The top region between the top electrode and the walls consists mainly of electrolytic fluid, which was modelled using 12 cells in height, using a mesh grading in the vertical direction, causing the cells on top of the walls being four times smaller in the vertical direction compared to the cells at the top of the pixel.

2. The central region spanned by the pixel walls and the volume enclosed by them was modelled using 16 cells in height, giving a much higher resolution locally since this is where most of the dynamics takes place.

3. The bottom region underneath the pixel (dielectric solid) is modelled by 10 cells in height, so that it properly captures the electric field in the solid layer.

The fluid region is a combination of the top region and the volume between the walls of the middle region. The walls consist of the rest of the middle region. Along the pixel length, a symmetry boundary was used so that the full pixel consists of $48 \times 96 \times 40$ pixels of which only half is simulated. In principle, another symmetry boundary could be set up, but computations have not been too lengthy. In the simulations, the ratio of conductivities of oil versus electrolyte liquid is $\frac{\sigma_{\text {oil }}}{\sigma_{\text {aq }}}=7 \times 10^{-5}$, and the ratio of permittivities is $\frac{\varepsilon_{\text {oil }}}{\varepsilon_{\mathrm{aq}}}=6.1^{\sigma_{\mathrm{aq}}} \times 10^{-2}$. The 


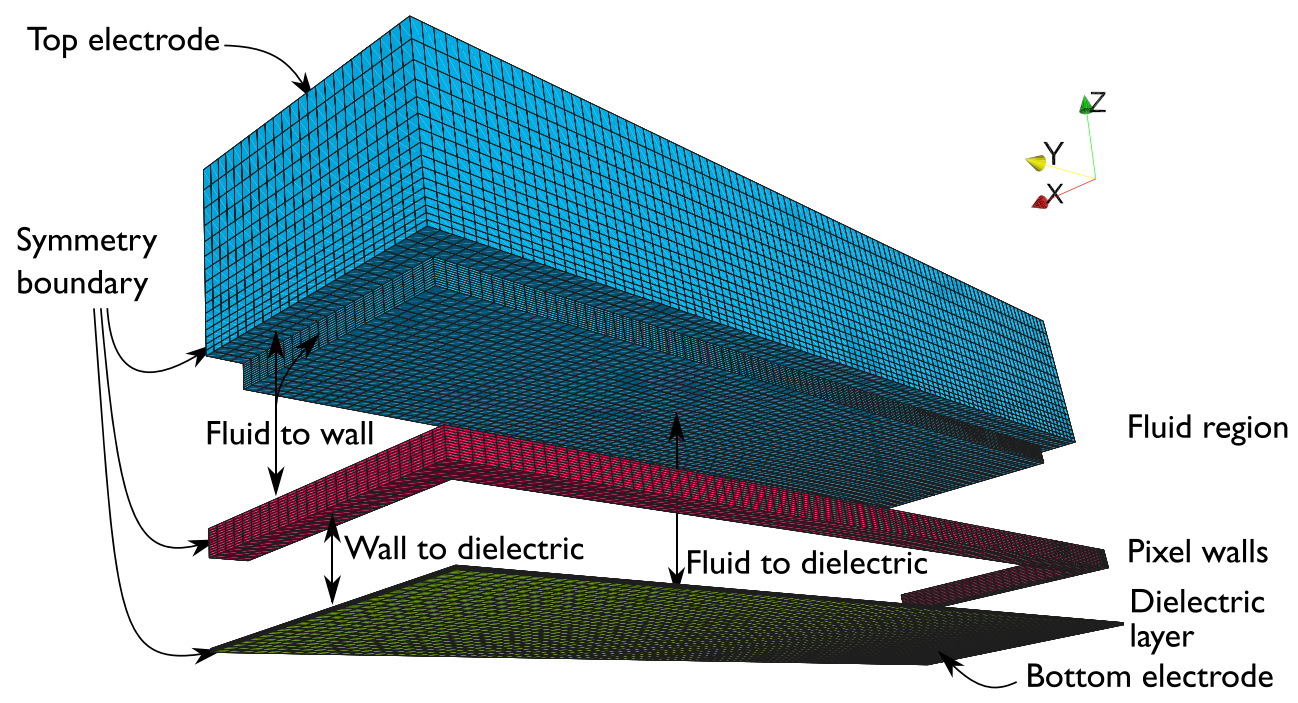

Fig. 9 Simulation mesh layout is shown, consisting of three different regions (walls, dielectric layer and fluid region) and their shared boundaries. The dielectric layer consists of ten cells in height, making the cells too fine to see in this figure

Table 3 Boundary conditions (BC) used for the different simulated variables

\begin{tabular}{|c|c|c|c|c|c|c|}
\hline & Phase fraction $\alpha$ & Velocity $\vec{u}$ & Pressure $p$ & Potential $\phi$ & Charge $\rho_{\mathrm{E}}$ & Description \\
\hline Top electrode & $\nabla \alpha=0$ & $\vec{u}=0$ & $\nabla p=0$ & $\phi=0 \mathrm{~V}$ & $\nabla \rho_{\mathrm{E}}=0$ & Impermeable wall with ground potential \\
\hline Bottom electrode & N/a & N/a & $\mathrm{N} / \mathrm{a}$ & $\phi=\phi_{\mathrm{V}} \mathrm{V}$ & N/a & $\begin{array}{l}\text { Predefined potential, not adjacent to fluid } \\
\text { region }\end{array}$ \\
\hline Fluid to dielectric & Contact angle $\mathrm{BC}$ & $\vec{u}=0$ & Fixed flux pressure & Mixed & $\nabla \rho_{\mathrm{E}}=0$ & Impermeable wall with coupled potential \\
\hline Fluid to wall & Contact angle $\mathrm{BC}$ & $\vec{u}=0$ & Fixed flux pressure & Mixed & $\nabla \rho_{\mathrm{E}}=0$ & Impermeable wall with coupled potential \\
\hline Wall to dielectric & N/a & N/a & N/a & Mixed & N/a & Solid-solid BC with coupled potential \\
\hline Outer domain BC & $\nabla \alpha=0$ & $\nabla \vec{u}=0$ & $\nabla p=0$ & $\nabla \phi=0$ & $\nabla \rho_{\mathrm{E}}=0$ & $\begin{array}{l}\text { Coupling to neighbour pixels, free fluid } \\
\text { in-outflow }\end{array}$ \\
\hline
\end{tabular}

The contact angle boundary condition is explained in Sect. 2.4 using a default (non-moving) contact angle of $\theta=60^{\circ}$, the mixed boundary condition is discussed in Sect. 2.3 and fixed flux pressure is a default OpenFOAM BC that keeps the pressure such that the zero gradient on velocity can be maintained

dielectric solid layer has an electric permittivity of $\varepsilon_{0} \varepsilon_{\mathrm{d}}=1.77 \times 10^{-11} \mathrm{~V} / \mathrm{m}$, and the walls have an electric permittivity of $\varepsilon_{0} \varepsilon_{\mathrm{d}}=3.54 \times 10^{-11} \mathrm{~V} / \mathrm{m}$. The general procedure is to set up the mesh and to initialize the oil into the pixel. The reference amount of oil is chosen such that when the oil completely covers the bottom boundary in between the walls, it is everywhere as high as the pixel walls, as sketched in the middle panel of Fig. 11. This means that when the oil is in de-wetting state, it curves up to be higher than the pixel walls, but due to contact line pinning to the sharp corners of the walls it remains constrained within the pixel geometry.

First, pixel closing dynamics is investigated, which is a process purely driven by capillarity. The volume of oil used inside a single pixel is varied, and the response times (from opened state to closed state) are evaluated. Subsequently, the pixel opening is studied, which is driven by an imposed voltage.

\subsection{Pixel closing}

The pixel closing dynamics (Fig. 10) is investigated from an initially stationary situation where the oil is contracted in a single corner. This situation is created by initializing an oil volume as a block on one side of the pixel using a bottom patch with a contact angle of $5^{\circ}$ (the rest of the bottom boundary was set to $60^{\circ}$ ) with respect to the oil phase. This makes the oil snap to the walls and stabilize, before the entire bottom is set to a $5^{\circ}$ static contact angle. Although this situation is somewhat artificial, this provides the most reproducible initial condition to compare the effect of the oil volume used inside a pixel. For the closing simulations, we use the original interFoam solver (i.e. not using the electric equations).

The area not covered by the oil, termed white area, is measured over time. As the oil spreads and covers a larger part of the bottom, several stages can be distinguished. In 


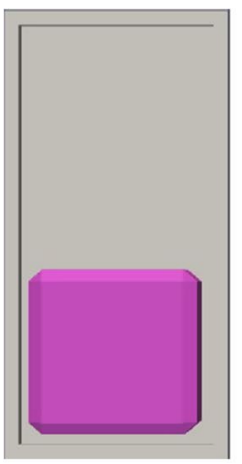

(a)

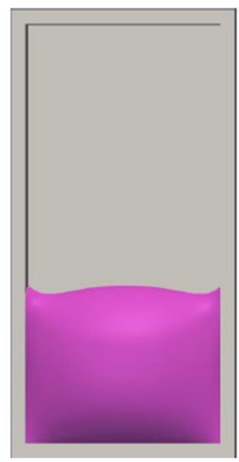

(b)

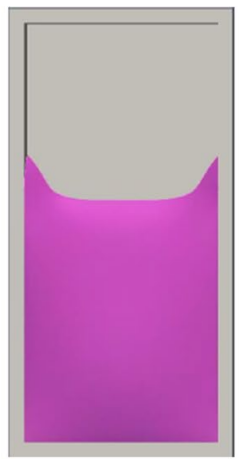

(c)

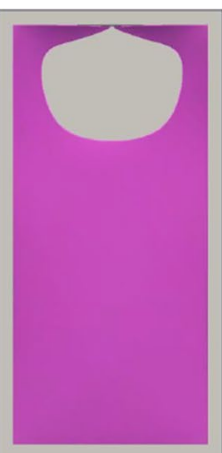

(d)

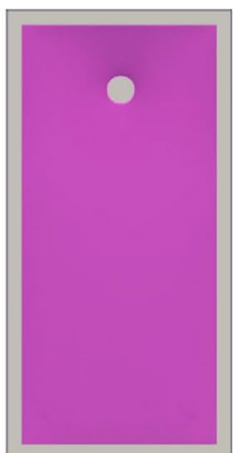

(e)

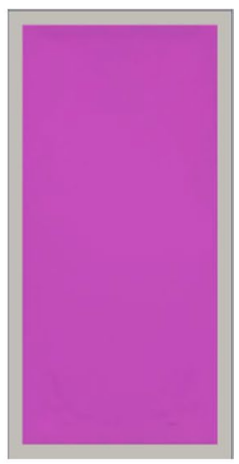

(f)
Fig. 10 Pixel closing simulations follow generally the given sequence; first, oil is initialized on one side of the domain, where it snaps to the nearby walls. The contact angle is then set to $5^{\circ}$ over the entire bottom; hence, oil will spread; first by bulk, then by capillary forces through the corners near the walls until these strands meet on the opposite side. The resulting circular area remains to close, which is typically the slow part of the process
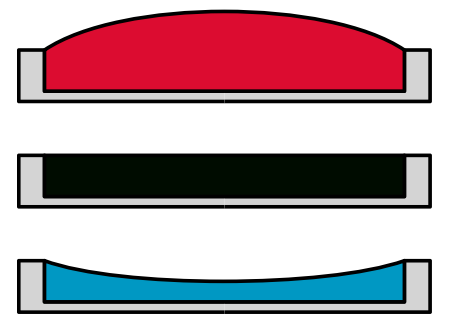

(a)

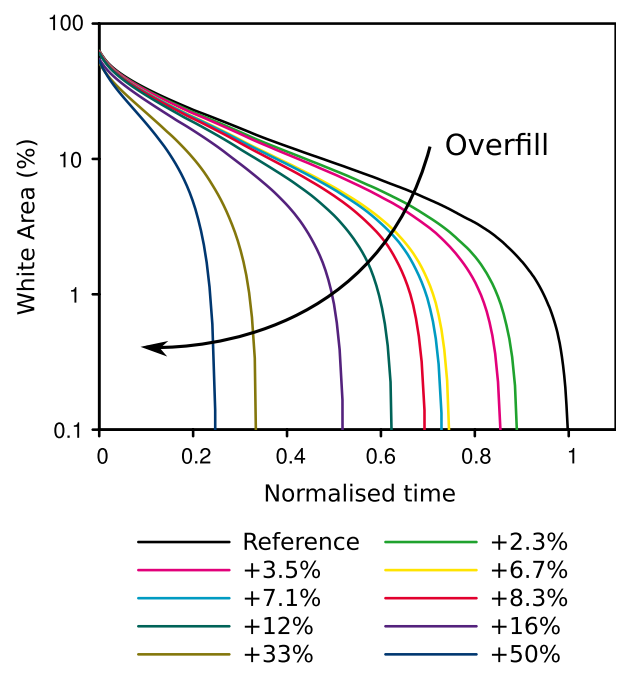

(b)

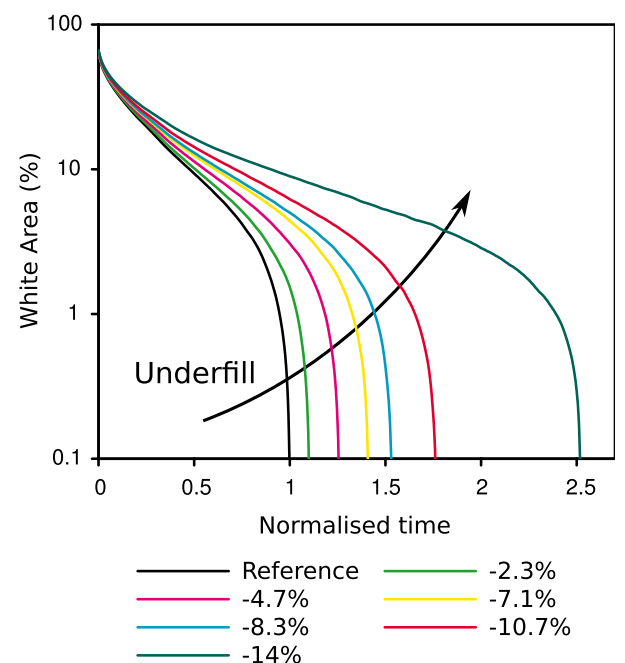

(c)
Fig. 11 Pixel closing simulations of the effect of overfilling [top in (a)] or underfilling [bottom in (a)] the oil volume in the pixel, and compared to the reference case [middle in (a)]. The semi-logarith-

the first stage, the bulk of the oil flows forward through the pixel, up to stage two where capillary effects take over and the oil flows along the left and right pixel walls until they meet on the opposite pixel side. The third stage starts with the covering of the circular area that has been left open, being the slowest part of the pixel closing (Fig. 10). While many simulations were performed using this technique, varying physical properties such as viscosity, surface tension, but also contact angle, pixel height, presence and size of satellite droplets (which may emerge due to thin film instability), aspect ratio of the pixel, in Fig. 11 the results are given for the oil filling level so that the effect of the oil volume can be quantified. Taking a reference case where the oil is mic graphs show the white area in percentage of the total pixel area, which decreases as a function of time due to oil spreading. Time is normalized by a reference closing time $t_{\text {ref }}=83 \mathrm{~ms}$

flat filled (when the oil layer completely covers the bottom, it is as high as the surrounding walls), the amount of oil can also be over-filled or under-filled, and the effect on the closing time is measured. It can be seen that the effect of oil volume has a more pronounced effect for the underfilling cases; a $14 \%$ smaller oil volume than the reference case increases the pixel response time with a factor slightly over 2.5 , while $16 \%$ more oil decreases the closing time no more than a factor of 2. While a faster response time is desired, a larger oil volume also causes a smaller initial white area.

The graphs are displayed on semilogarithmic scale to emphasize the different closing stages. While the initial part of the graphs shows a quick decrease in white area (the 
bulk stage), the lion's share of the response time is depicted linear meaning an exponentially decaying visible white area.

\subsection{Pixel opening}

Pixel opening is studied using the same geometry as used in the pixel closing case; a rectangular confinement with aspect ratio $2: 1$ surrounded by walls with a height of $4 \mu \mathrm{m}$, containing an amount of oil such that the volume between the walls is flat filled. The top of the domain contains the zero volt electrode, while the bottom electrode (underneath the dielectric layer) is set to a constant voltage. Both electrodes cover the entire pixel width and length, so that a symmetrical opening behaviour is anticipated. The bottom electrode is separated from the fluid domain by a dielectric layer, the thickness of which is set to $500 \mathrm{~nm}$ (a typical value for electrowetting purposes). The simulations are initialized at zero voltage for a number of time steps $(5 \mathrm{~ms})$ to allow for the fluid-fluid interface to reach an equilibrium position. This position is not exactly flat; this effect emerges from the fact that the oil-electrolyte interface is pinned at the sharp corner $\left(90^{\circ}\right.$ angle) of the walls, of which both the top side and the vertical (innerpixel) side have a defined contact angle. This results in a slight upward curved interface at the walls (which causes a slightly lower oil interface in the pixel centre). This initial oil interface height above the dielectric layer as it results from the simulation is shown in Fig. 12. After this initialization step, the simulation is restarted with the voltage immediately applied to the bottom electrode at full strength. The opening voltage has been varied between 15,20 and $25 \mathrm{~V}$, where they are compared to experiments, which have been performed by high-speed imaging of pixels with identical properties in terms of geometry and

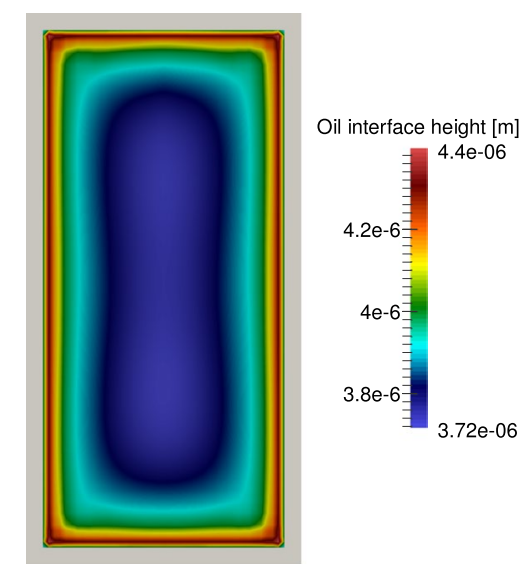

Fig. 12 Initial oil-electrolyte interface height in the pixel after zerovoltage initialization (electro)physical properties. The comparison is based on feature matching, as the exact experimental times when the voltage is applied could not be recorded simultaneous to the imaging. We have therefore matched the onset of the touchdown stage and related the simulation and experimental results from this point. The results are shown in Figs. 13, 14 and 15. First, the $15 \mathrm{~V}$ case is discussed in detail. After applying the voltage, this simulation takes about $19 \mathrm{~ms}$ to reach fully opened state. Different stages can be identified in the opening process.

1. Interface deflection. This stage is fully electrohydrodynamic (i.e. no electrowetting effects), as the threephase contact line remains pinned at the top of the pixel walls. Within the pixel, oil separates the aqueous phase from the dielectric layer everywhere. Charges build up at the fluid-fluid interface and the Maxwell stress pulls the interface towards the bottom electrode. The oil film progressively thins during this stage throughout the central area of the pixel. This process is the same as for electrowetting-functionalized superhydrophobic surface (Manukyan et al. 2011) and electrically tunable optical apertures (Murade et al. 2011).

2. Water-dielectric contact formation. The oil-water interface reaches the dielectric layer and the oil film breaks up (Staicu and Mugele 2006). This leads to the formation of a three-phase contact line-possibly including a molecularly thin residual oil layer that is not resolved with the present simulation technique. Charge accumulates at the oil cleared area, which restrict the electric field to the dielectric layer only.

3. Expansion. The oil moves to the pixel sides forming two bodies at either side of the pixel. The electric field is strong on the oil side of the moving contact line which is the driving force for the retraction of the oil.

4. Relaxation. The contact line of the bulk stops moving, but the oil in the small filaments along the pixel side corners is still flowing to the bulk. During the simulation, small amounts of oil have been left on the pixel bottom, fractions that are lower than the visualization threshold requires to draw an interface (recall that the VOF method used is a numerical technique that smears the interface), which gather in the cells in the pixel centre. This process is affected by deficiencies of the smeared interface. We are therefore unable to assess the quantitative accuracy of the behaviour of these satellite drops. Note, however, that the occurence of satellite drops is physical. They appear in the present experiments (see Figs. 13, 14, 15), and they were observed before (Murade et al. 2011; Staicu and Mugele 2006; Sun and Heikenfeld 2008). Using stability analysis in lubrication approximation of thin oil films between an electrode and a water film, it is possible to show that 


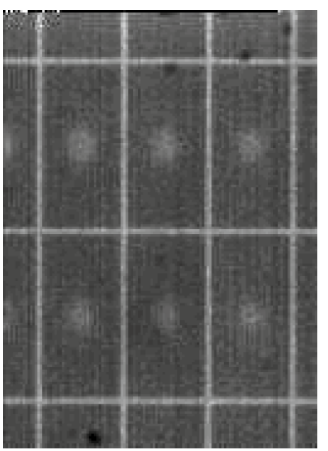

(a)

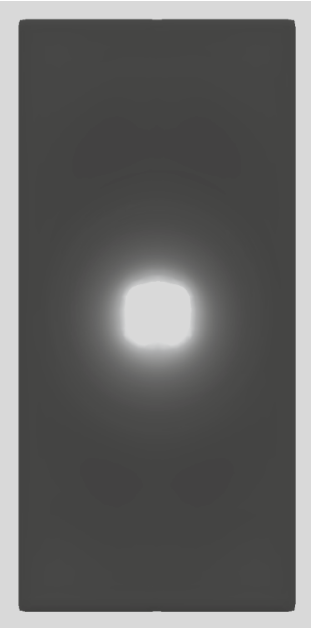

(e)

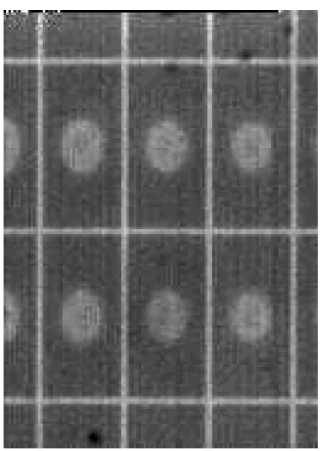

(b)

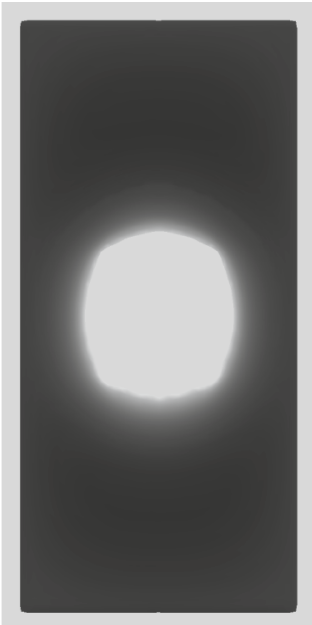

(f)

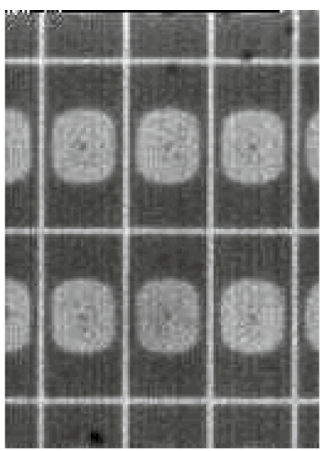

(c)

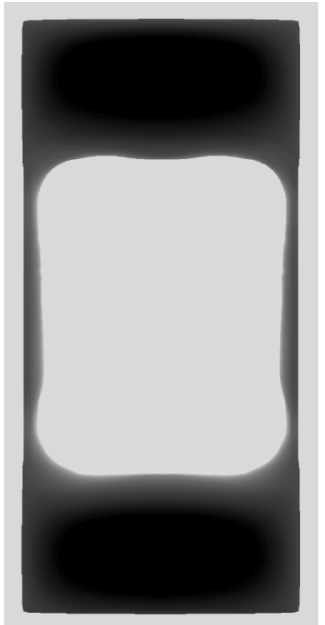

(g)

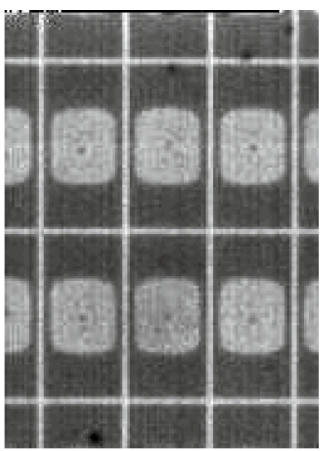

(d)

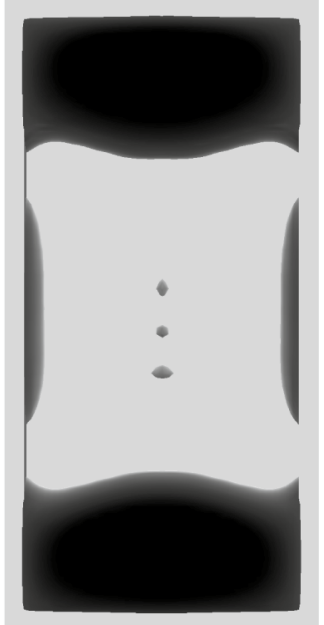

(h)
Fig. 13 Pixel opening at $15 \mathrm{~V}$, where the top row of images displays experimental results of several pixels and the bottom row the simulation of a single pixel. Different stages include interface deflection,

satellite drops form due to a linear instability (Staicu and Mugele 2006).

The dynamic response of the simulated oil is compared to the experimental results. In order to synchronize the two data series, the moment of water-dielectric contact formation is matched, from which the other frames can be compared in absolute time scales. It can be seen that the transient effect of pixel opening is perfectly captured by the simulations. A few small mismatches in oil pattern can be observed, most notably the convex bending of the oil bulk in the simulations, whereas the oil profile is concave in the experiments.

Comparing Figs. 13, 14 and 15, it is clear that the oil withdrawal behaviour is strongly dependent on the voltage applied; both the distribution of the oil and the absolute time of pixel opening vary substantially. A distinctive feature of the $25 \mathrm{~V}$ case (and to some extend for the $20 \mathrm{~V}$ case touchdown, expansion and relaxation (capillary flow). Times are normalized with respect to their ending time a $t=0.053$, b $t=0.132$, c $t=0.474, \mathbf{d} t=1.0, \mathbf{e} t=0.053, \mathbf{f} t=0.132, \mathbf{g} t=0.474, \mathbf{h} t=1.0$

as well) is that the interface deflection takes place at two places instead of in the pixel centre. This results in an oil bulk at the pixel side walls in the final state, which in the simulation eventually lies on top of the walls.

Minor differences between experiments and simulations may occur for several reasons. First of all, the conductivity of the fluids may not be constant, especially if the pixels are actuated a lot. The simulation assumes insolubility of the two phases, but in reality a very small amount of solubility or dispersion of one fluid in the other may occur, although this has not been verified by experiments. Also, a closer investigation of the dielectric layers and pixel bottoms of other samples has shown that varying dielectric layer thickness, as well as differences in the pixel depth, may occur, which of course may influence the moment of touch-down and final shape of the interface. This is another reason why feature matching has been used to compare simulations and experiments from the moment of touchdown onward, since after the moment of touch-down the 


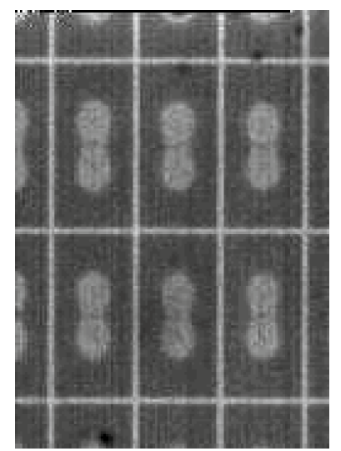

(a)

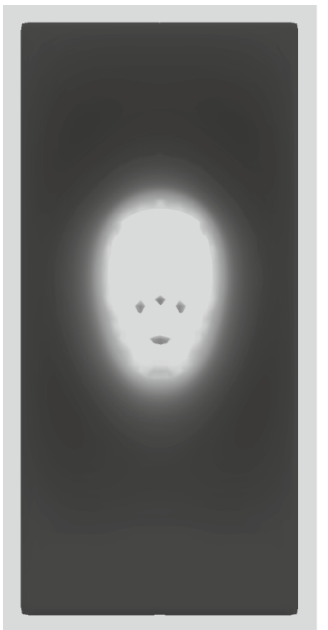

(e)

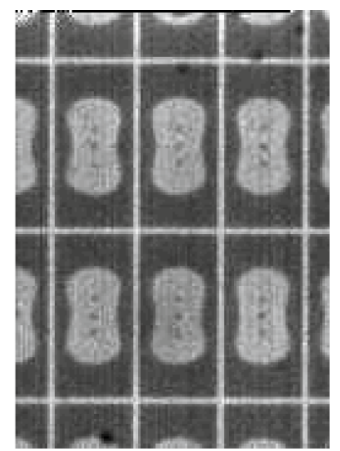

(b)

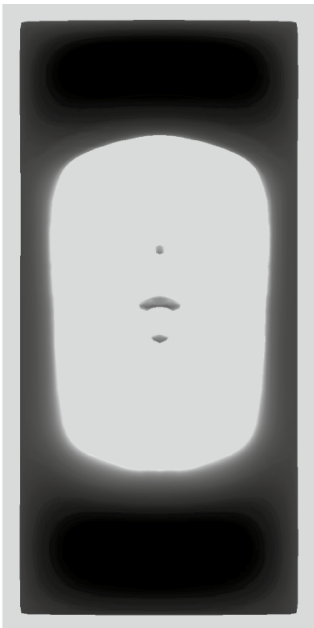

(f)

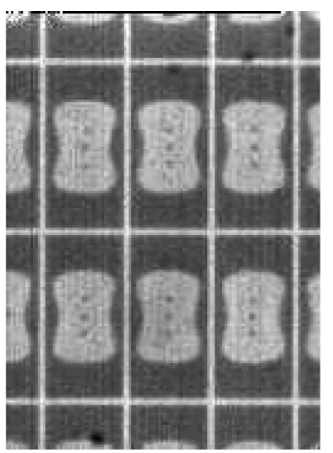

(c)

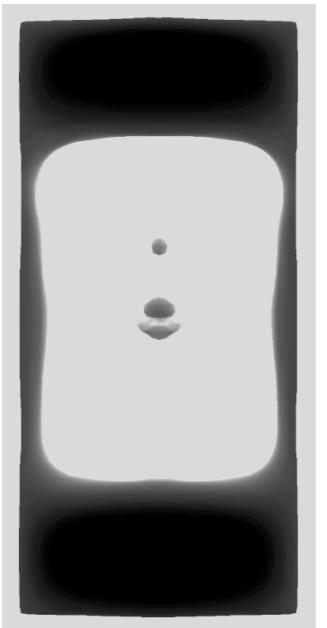

(g)

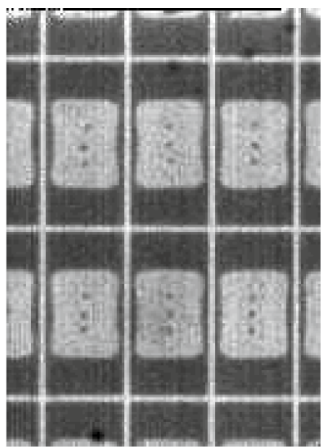

(d)

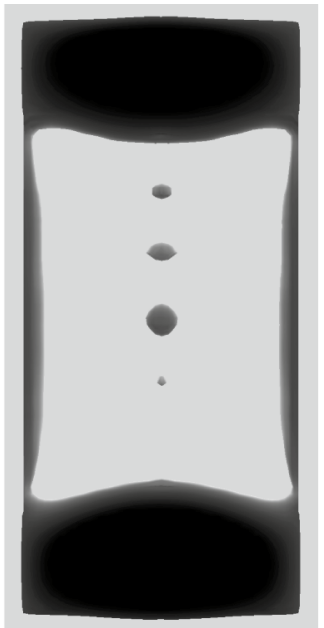

(h)
Fig. 14 Pixel opening at $20 \mathrm{~V}$, where the top row of images displays experimental results of several pixels and the bottom row the simulation of a single pixel. The distinctive fluid layer on the side walls is

dynamics depend much less on variations in dielectric layer thickness or pixel bottom height.

Moreover, it should be noted that the final dynamics of break-up of the oil layer, when the interface is only 1 grid cell thick, cannot be captured by our CFD simulations. Yet, the overall agreement between our simulations and the experiments suggests that these effects have a minor influence on the global dynamics of pixel opening.

Experiments on EWOD devices require a dielectric layer to be present between the electrode and the fluid phases to prevent electrolysis. Yet, using the numerical model, it is possible to perform a simulation without a dielectric layer (i.e. using the bottom boundary of the fluid flow domain as electrode with a constant electric potential). The lack of a dielectric layer essentially increases the electrostatic force on the fluid-fluid surface as compared to a simulation (or experiment) that does include the dielectric layer. We have observed that a simulated pixel opening corresponds to a higher-voltage experiment that does include a dielectric layer. Hence, a reliable, accurately captured. Times are normalized with respect to their ending time. a $t=0.07$, b $t=0.29$, c $t=0.43$, d $t=1.0$, e $t=0.07$, f $t=0.29, \mathbf{g} t=0.43, \mathbf{h} t=1.0$

quantitative result can only be obtained when taking the dielectric layer into account.

\subsection{Mesh dependency}

We have performed a mesh dependency study with the $20 \mathrm{~V}$ pixel opening case into the quality of the results with different mesh sizes (see Fig. 16). The base case, as explained in the previous section, consists of a total of $24 \times 96 \times 38$ cells, using a symmetry boundary condition along the long axis of the pixel. Keeping the cells similarly sized, we have also performed the same simulations with a more coarse $(18 \times 72 \times 29)$ and a more fine mesh $(36 \times 144 \times 57)$.

It was found that the coarse mesh produces a much faster touch-down stage (recall that numerical interface break-up happens faster with larger cells), but evolves into the similar shapes eventually. The base case and finer meshes produce very similar results, based on visual inspection of the simulation time steps. The touch-down event occurs on a very comparable time step (with only $20 \mu$ s in between, 


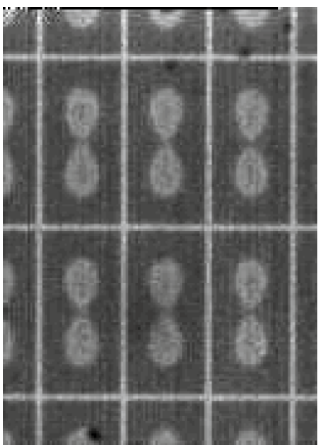

(a)

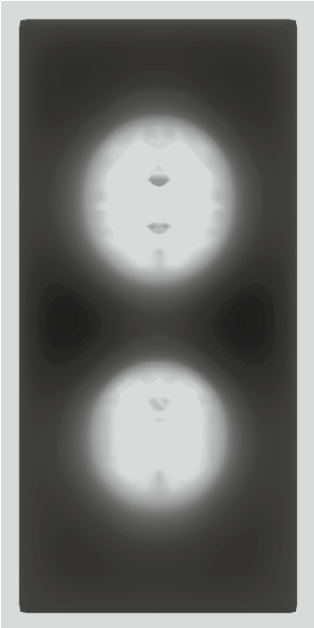

(e)

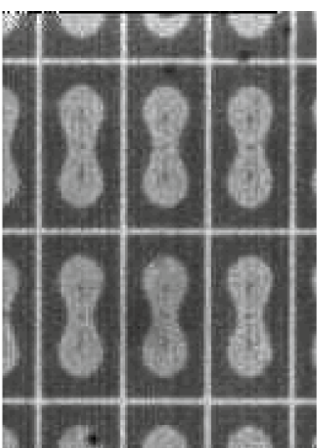

(b)

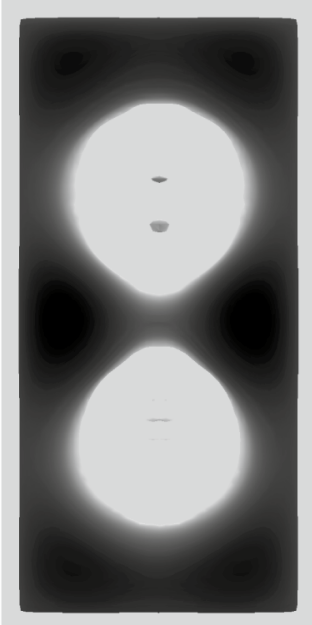

(f)

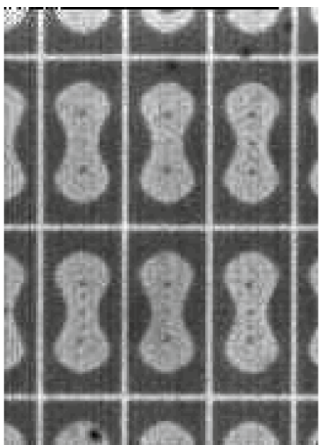

(c)

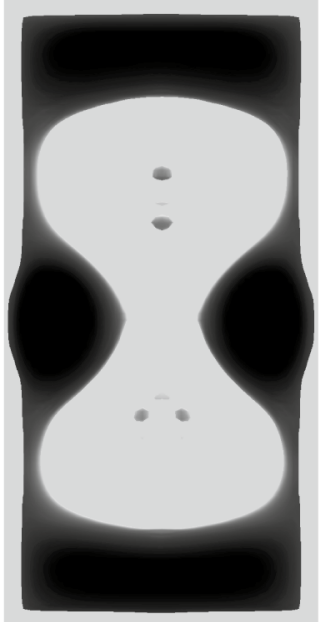

(g)

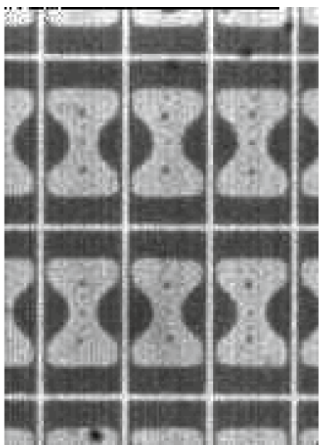

(d)

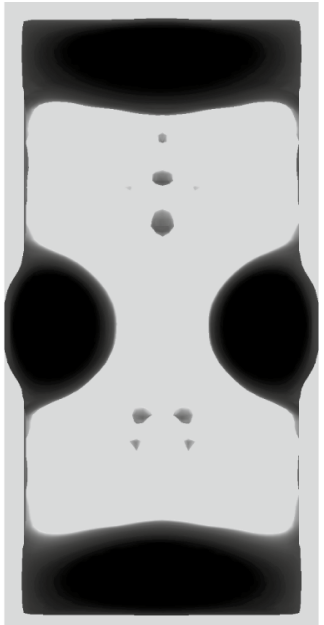

(h)
Fig. 15 Pixel opening at $25 \mathrm{~V}$, where the top row of images displays experimental results of several pixels and the bottom row the simulation of a single pixel. Different stages include interface deflec- tion, touchdown, expansion, relaxation and capillary flow. Times are normalized with respect to their ending time. a $t=0.1 \mathbf{b} t=0.21 \mathbf{c}$ $t=0.40 \mathbf{d} t=1.0 \mathbf{e} t=0.1 \mathbf{f} t=0.21 \mathbf{g} t=0.40 \mathbf{h} t=1.0$
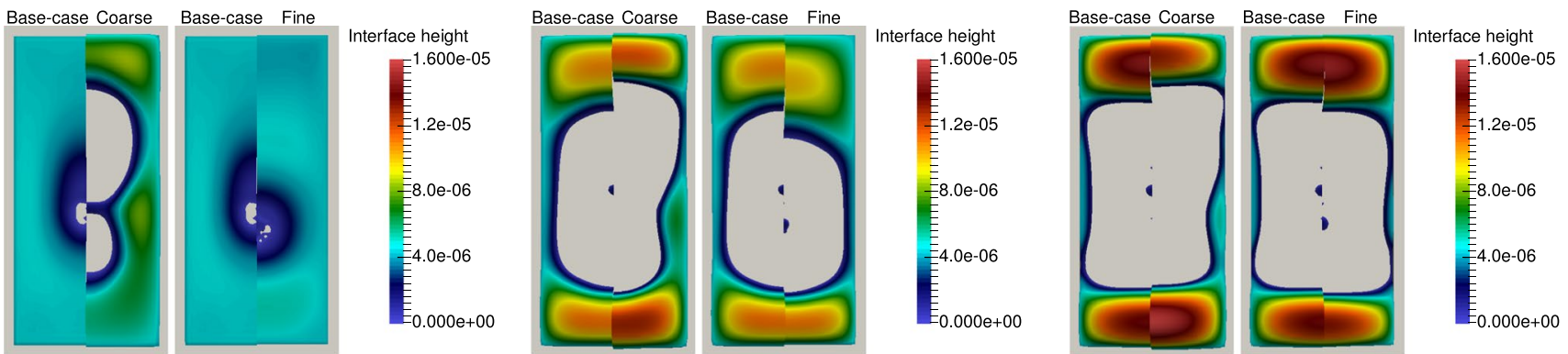

Fig. 16 Simulation snapshots at different stages of pixel opening at $20 \mathrm{~V}$. The coarse mesh reaches the touch-down stage much earlier than the default (base case) and refined mesh. The fine mesh and base case produce very similar results

a fraction of the total of $19 \mathrm{~ms}$ required for opening the pixel). The absence of symmetry is somewhat distracting and is caused by a non-symmetrical initial position of the interface. Eventually, the simulation reaches a symmetrical final state.

The white area is one of the most important characteristics of the pixels, during opening and closing behaviour, and is quantifiable using the visualization of the interface. However, the comparison between mesh sizes has been made in a qualitative way only, since the occurence of satellite droplets (due to thin film instability) occurs differently for different mesh sizes. In actual pixels, thin films/ droplets are translucent, but in the simulations, they do block the pixel bottom. For this reason, different mesh sizes 
are not quantitatively comparable, and the comparison has been done in a qualitative way, similar to our comparison with experiments.

\section{Discussion and conclusion}

An electrohydrodynamic model for the simulation of electrowetting on dielectric devices has been implemented and described in this work. The current implementation is based on the OpenFOAM framework, using a volume of fluid method to account for the different fluid phases. Via a charge transport equation and Gauss's law, electrostatic field calculations are performed based on a hydrodynamically resolved fluid-fluid interface, and the electric force acting on the fluid-fluid interface is taken into account. Furthermore, a multi-region approach has been employed to simulate the effect of a solid dielectric layer that is impermeable for the fluids and electric charges, but takes into account the electric field distribution.

Different aspects of the model have been verified using a number of synthetic benchmark cases, after which the model has been used to simulate the closing and opening behaviour of display pixels based on electrowetting. The pixels employ a transparent aqueous phase and an opaque oil phase which are actuated via an applied electric potential. Pixel closing has been studied by initializing an amount of oil on the side of the pixel, which spreads out over the pixel bottom. The effect of different oil volumes on the closing time and on the uncovered area has been described, along with a discussion of different stages of the closing behaviour.

Pixel opening behaviour has been simulated and compared to experiments. At three voltages, 15,20 and $25 \mathrm{~V}$, the simulations show a very good correspondence of the whole dynamics with measurements. Again, a number of different stages in opening behaviour have been described.

Several improvements of the algorithm can be considered. First of all, the VOF method as used in this work is not ideal, since the smeared interface may cause for instance spurious currents and (potentially artificial) satellite droplets. The impact of these aspects is small for the situations studied here. Further improvements could therefore be achieved by incorporation of geometric interface reconstruction (e.g. Maric et al. 2013). Additionally, a more accurate calculation of the interface curvature can be achieved by using height functions for curvature calculations (Afkhami and Bussmann 2009; Popinet 2009). These methods are at this point not incorporated in the public version of OpenFOAM.

The current implementation of the solid-to-fluid domain boundary conditions mimics, but is not quite the same as, the actual physical description of coupling the electric potential between domains. First of all, the electric potential value of the solid domain $\left(\phi_{\mathrm{s}}\right)$ and fluid flow domain $\left(\phi_{\mathrm{f}}\right)$ should match:

$\phi_{\mathrm{s}}=\phi_{\mathrm{f}}$

Secondly, the gradients are coupled via the ratio of electric permittivities:

$\varepsilon_{\mathrm{s}} \frac{\partial \phi_{\mathrm{s}}}{\partial n}=\varepsilon_{\mathrm{f}} \frac{\partial \phi_{\mathrm{f}}}{\partial n}$

OpenFOAM does not (directly) offer a boundary condition that meets these requirements. We have, however, chosen to use the boundary condition as described before (Eq. 19), which performs very well in this scenario.

Acknowledgments This work is part of the VICI research programme 'Switchable Superhydrophobic Surfaces', which is financed by the Netherlands Organization for Scientific Research (NWO) and the Dutch Technology Foundation (STW).

Conflict of interest The authors declare that they have no conflict of interest.

Open Access This article is distributed under the terms of the Creative Commons Attribution 4.0 International License (http://creativecommons.org/licenses/by/4.0/), which permits unrestricted use, distribution, and reproduction in any medium, provided you give appropriate credit to the original author(s) and the source, provide a link to the Creative Commons license, and indicate if changes were made.

\section{References}

Afkhami S, Bussmann M (2009) Height functions for applying contact angles to $3 \mathrm{~d}$ vof simulations. Int $\mathrm{J}$ Numer Methods Fluids 61:827-847

Aminfar H, Mohammadpourfard M (2009) Lattice Boltzmann method for electrowetting modeling and simulation. Comput Methods Appl Mech Eng 198:3852-3868

Aminfar H, Mohammadpourfard M (2012) Droplets merging and stabilization by electrowetting: Lattice Boltzmann study. J Adhes Sci Technol 26:1853-1871

Arzpeyma A, Bhaseen S, Dolatabadi A, Wood-Adams P (2008) A coupled electro-hydrodynamic numerical modeling of droplet actuation by electrowetting. Colloids Surf A: Physicochem Eng Asp 323:28-35

Baltussen MW, Kuipers JAM, Deen NG (2014) A critical comparison of surface tension models for the volume of fluid method. Chem Eng Sci 109:65-74

Berge B, Perseux J (2000) Variable focal lens controlled by an external voltage: an application of electrowetting. Eur Phys J E 3:159-163

Bjørklund E (2009) The level-set method applied to droplet dynamics in the presence of an electric field. Comput Fluids 38:358-369

Bonn D, Eggers J, Indekeu J, Meunier J, Rolley E (2009) Wetting and spreading. Rev Mod Phys 81:739-805

Brackbill JU, Kothe DB, Zemach C (1992) A continuum method for modeling surface tension. J Comput Phys 100:335-354 
Buehrle J, Herminghaus S, Mugele F (2003) Interface profiles near three-phase contact lines in electric fields. Phys Rev Lett 91:086101

Clime L, Brassard D, Veres T (2010a) Numerical modeling of electrowetting processes in digital microfluidic devices. Comput Fluids 39:1510-1515

Clime L, Brassard D, Veres T (2010b) Numerical modeling of electrowetting transport processes for digital microfluidics. Microfluid Nanofluidics 8:599-608

Cox RG (1986) The dynamics of the spreading of liquids on a solidsurface. 1. Viscous-flow. J Fluid Mech 168:169-194

Deshpande SS, Anumolu L, Trujillo MF (2012) Evaluating the performance of the two-phase flow solver interfoam. Comput Sci Discov 5:014016

Dijkhuizen W, Roghair I, Van Sint Annaland M, Kuipers JAM (2010) Dns of gas bubbles behaviour using an improved 3d front tracking model-model development. Chem Eng Sci 65:143-1427

Dolatabadi A, Mohseni K, Arzpeyma A (2006) Behaviour of a moving droplet under electrowetting actuation: numerical simulation. Can J Chem Eng 84:17-21

Drygiannakis AI, Papathanasiou AG, Boudouvis AG (2009) On the connection between dielectric breakdown strength, trapping of charge, and contact angle saturation in electrowetting. Langmuir $25: 147-152$

Fair RB (2007) Digital microfluidics: is a true lab-on-a-chip possible. Microfluid Nanofluid 3(3):245-281

Hayes RA, Feenstra BJ (2003) Video-speed electronic paper based on electrowetting. Nature 425:383-385

Hirt CW, Nichols BD (1981) Volume of fluid (vof) method for the dynamics of free boundaries. J Comput Phys 39:201-225

Hoang DA, van Steijn V, Portela LM, Kreutzer MT, Kleijn CR (2013) Benchmark numerical simulations of segmented two-phase flows in microchannels using the volume of fluid method. Comput Fluids 86:28-36

Hong JS, Ko SH, Kang KH, Kang IS (2008) A numerical investigation on ac electrowetting of a droplet. Microfluid Nanofluidics 5:263-271

Jones TB, Gunji M, Washizu M, Feldman MJ (2001) Dielectrophoretic liquid actuation and nanodroplet formation. J Appl Phys 89:1441

Keshavarz-Motamed Z, Kadem L, Dolatabadi A (2010) Effects of dynamic contact angle on numerical modeling of electrowetting in parallel plate microchannels. Microfluid Nanofluidics 8:47-56

Krupenkin T, Taylor JA (2011) Reverse electrowetting as a new approach to high-power energy harvesting. Nat Commun 2:101038

Krupenkin T, Yang S, Mach P (2003) Tubable liquid microlens. Appl Phys Lett 82:316-318

Ku Y-S, Kuo S-W, Huang Y-S, Chen C-Y, Lo K-L, Cheng W-Y, Shiu J-W (2011) Single-layered multi-color electrowetting display by using ink-jet-printing technology and fluid-motion prediction with simulation. J SID 19(7):488-495

Kuiper S, Hendriks BHW (2004) Variable-focus liquid lens for miniature cameras. Appl Phys Lett 85:1128-1130

Le Grand Nolwenn, Daerr Adrian, Limat Laurent (2005) Shape and motion of drops sliding down an inclined plane. J Fluid Mech 541:293-315

Lima NC, d'Ávila MA (2013) Electric effects on conducting newtonian and viscoelastic droplets. In: 22nd International Congress of Mechanical Engineering, COBEM, Ribeirão Preto, SP, Brazil

López-Herrera JM, Popinet S, Herrada MA (2011) A charge-conservative approach for simulating electrohydrodynamic two-phase flows using volume-of-fluid. J Comput Phys 230:1939-1955

Manukyan G, Oh JM, van den Ende D, Lammertink RGH, Mugele F (2011) Electrical switching of wetting states on superhydrophobic surfaces: a route towards reversible Cassie-toWenzel transitions. Phys Rev Lett 106:014501

Maric T, Marschall H, Bothe D (2013) voFoam-a geometrical volume of fluid algorithm on arbitrary unstructured meshes with local dynamic adaptive mesh refinement using OpenFOAM. ArXiv e-prints

Mugele F, Baret J-C (2005) Electrowetting: from basics to applications. J Phys: Condens Matter 17:R705-R774

Mugele F, Buehrle J (2007) Equilibrium drop surface profiles in electric fields. J Phys: Condens Matter 19:375112

Murade CU, van der Ende D, Mugele F (2012) High speed adaptive liquid microlens array. Opt Express 20:18180-18187

Murade CU, Oh JM, van den Ende D, Mugele F (2011) Electrowetting driven optical switch and tunable aperture. Opt Express 19:15525

Muzaferija S, Perić M (1998) Computation of free surface flows using interface-tracking and interface-capturing methods. In: Mahrenholtz O, Markiewicz M (eds) Nonlinear water wave interaction. Computational Mechanics Publications, Southampton

Oh JM, Legendre D, Mugele F et al (2012) Shaken not stirred-on internal flow patterns in oscillating sessile drops. Eur Phys Lett 98:34003

Pooyan S, Passandideh-Fard M (2012) On a numerical model for free surface flows of a conductive liquid under an electrostatic field. J Fluids Eng 134:091205

Popinet S (2009) An accurate adaptive solver for surface-tensiondriven interfacial flows. J Comput Phys 228:5838-5866

Roghair I, van den Ende HTM, Mugele F (2013) An openfoam-based electro-hydrodynamic model. In: 8th International Conference on Multiphase Flow, Jeju, Korea

Saville DA (1997) Electrohydrodynamics: the Taylor-Melcher leaky dielectric model. Annu Rev Fluid Mech 29:27-64

Snoeijer JH, Andreotti B (2013) Moving contact lines: scales, regimes, and dynamical transitions. Annu Rev Fluid Mech 45:269-292

Staicu A, Mugele F (2006) Electrowetting-induced oil film entrapment and instability. Phys Rev Lett 97:167801

Sun B, Heikenfeld J (2008) Observation and optical implications of spinodal dewetting in electrowetting displays. J Micromech Microeng 18:025027

Sussman M, Smereka P, Osher S (1994) A level set approach for computing solutions to incompressible two-phase flow. J Comput Phys 114:146-159

Sussman M, Almgren AS, Bell JB, Colella P, Howell LH, Welcome ML (1999) An adaptive level set approach for incompressible two-phase flows. J Comput Phys 148:81-124

Taylor G (1966) Studies in electrohydrodynamics: I-the circulation produced in a droplet by electric field. Proc R Soc Lond Ser A: Math Phys Sci 291:159-166

Tomar G, Gerlach D, Biswas G, Alleborn N, Sharma A, Durst F, Welch SWJ, Delgado A (2007) Two-phase electrohydrodynamic simulations using a volume-of-fluid approach. J Comput Phys 227:1267-1285

Ubbink O, Issa RI (1999) Method for capturing sharp fluid interfaces on arbitrary meshes. J Comput Phys 153:26-50

Unverdi SO, Tryggvason G (1992) A front-tracking method for viscous, incompressible, multi-fluid flows. J Comput Phys 100:25-37

Voinov OV (1976) Hydrodynamics of wetting. Fluid Dyn 11:714-721

Zalesak ST (1979) Fully multidimensional flux-corrected transport algorithms for fluids. J Comput Phys 31:335-362 